\title{
Fourier mode analysis of layers in shallow shell deformations
}

\section{Report}

Author(s):

Pitkäranta, J.; Matache, Ana-Maria; Schwab, Christoph

Publication date:

1999-09

Permanent link:

https://doi.org/10.3929/ethz-a-004284974

Rights / license:

In Copyright - Non-Commercial Use Permitted

Originally published in:

SAM Research Report 1999-18 


\title{
Fourier mode analysis of layers in shallow shell deformations
}

\author{
J. Pitkäranta*, A.-M. Matache and C. Schwab
}

Research Report No. 99-18

September 1999

Seminar für Angewandte Mathematik

Eidgenössische Technische Hochschule

CH-8092 Zürich

Switzerland

${ }^{*}$ Institute of Mathematics, Helsinki University of Technology, Otakaari 1, SF-02150 Espoo, Finland 
Fourier mode analysis of layers in shallow shell deformations

J. Pitkäranta*, A.-M. Matache and C. Schwab

Seminar für Angewandte Mathematik

Eidgenössische Technische Hochschule

CH-8092 Zürich

Switzerland

Research Report No. 99-18 September 1999

\begin{abstract}
We investigate here the length scales of the boundary or interior layer effects in shell deformation. Quantitative information on the layers is obtained by considering two (simplified) 'shallow' shell models corresponding to the 'classical' three-field (Love-Koiter-Novozhilov), resp. five-field (Reissner-Naghdi) shell models.

We start by analysing the layers as functions of the thickness of the shell, while keeping the other geometric parameters fixed. Having found the four limit Fourier modes we complete the analysis investigating systematically the layers length scales under more general assumptions, particularly when also the wave parameter is variable. Scaling properly the energy expressions, as indicated by the layer mode analysis, shell deformation energies characteristic to shell layers are found. These show how the layer is effectively seen by the finite element solver and can be useful in the analysis of numerical locking effects in the FEM approximation of shell layers.
\end{abstract}

${ }^{*}$ Institute of Mathematics, Helsinki University of Technology, Otakaari 1, SF-02150 Espoo, Finland 


\section{Introduction}

In this paper we study the length scales of the boundary or interior layer effects in shell deformations. We proceed from two-dimensional linear shell models where the deformation of the shell is expressed in terms of a three- or five-component vector field defined on the midsurface of the shell. The vector field consists of the three displacements of the midsurface and, optionally, of two dimensionless rotations physically related to transverse shear deformations. Many variants of two-dimensional shell models of this kind are available in the wide literature on the engineering and mathematical theory of shells, as pioneered by Love [13, 14] and later contributed to by a large number of authors including Flügge [5, 6], Vlasov [35], Novozhilov [19], Gol'denveizer [7], E. Reissner [26, 27], Koiter [11, 12], Naghdi [16, 17] and many others, cf. also $[18,33]$ and the further references in the books cited. As starting points of this work we choose two particular models, a 'classical' three-field model named here LNK (after Love-Novozhilov-Koiter), and a parametrized five-field model to be named RN (after Reissner-Naghdi).

The motivation of the work comes from the numerical modelling of shells by finite elements, see $[24,9,10,8]$ and the further references therein. In the finite element modelling of shells, there arise various parametric error amplification or locking phenomena that are not well understood currently. Part of the problem is in the insufficient understanding of the asymptotic nature of shell deformation states from the numerical point of view. Here it is particularly important to understand the layer effects, since maximal stresses typically occur near boundaries, or junctions, or other irregularities where layers can be strong. That layers often are strong, and have wide ranges, is a characteristic feature of shell problems and shell deformation states. This in fact is one of the features that makes shell problems rather unique among problems in computational science.

For the needs of modern numerical analysis, the current knowledge of the layers in shell deformations is rather limited. The mathematical shell theory is quite brief on this subject, giving little beyond the pioneering discussion by Gol'denveizer [7] and the later, more ambitious but formal asymptotic analysis by Rutten $[28,29]$. In the engineering shell theory, important special cases like cylindrical shells have been treated quite extensively [6, 34], but otherwise the engineering layer theory is limited as well. Thus there is a need of both extending and modernizing the classical shell theory on the subject of layers, taking into account the reality of large-scale numerical modelling and the mathematical questions arising there. The present work is an attempt in that direction.

In order to obtain quantitative information on the layers, we make further simplifying geometric assumptions within the chosen LNK- and RN-models. The simplifications lead to two variants of approximate models that we label as $\mathrm{LNK}_{\mathrm{S}}, \mathrm{RN}_{\mathrm{S}}$ and $\mathrm{LNK}_{\mathrm{SS}}, \mathrm{RN}_{\mathrm{SS}}$ and call shallow shell models. By 'shallow' we mean that the dimensions of the shell are small compared with the principal curvatures of its midsurface. We define then $\mathrm{LNK}_{\mathrm{S}}$ and $\mathrm{RN}_{\mathrm{S}}$ as asymptotic models corresponding to the parametric limit $\epsilon=L / R \rightarrow 0$, where $L$ is the diameter (or largest dimension) of the midsurface and $R$ is the curvature length scale. We may view $L$ here alternatively as the length scale that characterizes the variation of the displacements 
and rotations along the midsurface, so the shallow shell approximation is reasonable when modelling local effects (such as layers) in length scales $L \ll R$.

Shallow shell models of various kinds have their roots in the engineering theory of shells $[35,6]$. Simpler models have been useful, e.g., in stability studies [35] or as a basis of analytic or approximate solution of the shell equations in specific situations, cf. [15]. In the important special case of a cylindrical shell the usual 2D shell equations take a 'shallow' form, so there is no need for geometric simplifications. Here we have $\mathrm{LNK}=\mathrm{LNK}_{\mathrm{S}}$ and $\mathrm{RN}=\mathrm{RN}_{\mathrm{S}}$ in that case, and the latter models remain approximately valid also for axially shallow (nearly cylindrical) shells of revolution.

In all non-asymptotic 2D models of classical linear shell theory, the shell equations are written as a system of equilibrium laws of the form

$$
\mathcal{M} \mathbf{u}+t^{2} \mathcal{B} \mathbf{u}=\mathbf{f} \text { on } \omega
$$

where $\omega$ is the midsurface of the shell, $\mathbf{u}$ is the displacement vector field to be found, $t$ is the thickness of the shell, and $\mathcal{M}, \mathcal{B}$ are linear partial differential operators that depend on the geometry of $\omega$, and on the material parameters, but are independent of $t$. Physically $\mathcal{M u}$ expresses the forces arising from the stretching and (in case of the RN-model) transverse shear deformations, and $t^{2} \mathcal{B} \mathbf{u}$ expresses the bending forces. Layers in the displacement field $\mathbf{u}$ arise basically because operator $\mathcal{B}$ is of higher order than $\mathcal{M}$ and thus causes a singular perturbation in (1.1) at small $t$. We call here a vector field $\mathbf{u}_{l}$ a layer if it has the following properties:

1. $\mathbf{u}_{l}$ is parametrized with $t$ and satisfies the homogeneous Eqs. (1.1) away from $S$, where $S$ is a subset of $\bar{\omega}$ of measure zero - called the layer generator.

2. $\mathbf{u}_{l}$ decays exponentially away from $S$, as follows: Let $P \in \omega, P \notin S$, and let $Q=$ $Q(P) \in \bar{S}$ be such that $d(P)=\operatorname{dist}(P, S)=|\overrightarrow{Q P}|$. Further let $\vec{\tau}$ be the unit vector parallel with $\overrightarrow{Q P}$. Then as $P$ varies, there exists $L=L(Q, \vec{\tau}, t)>0$ such that $\left|\mathbf{u}_{l}(P)\right| \sim$ $\exp \{-d(P) / L\}$.

3. When $t$ varies, the length scale $L$ above varies so that $L(Q, \vec{\tau}, t) \rightarrow 0$ as $t \rightarrow 0$.

In a given problem setup, the layer generator $S$ can be found by studying the properties of the asymptotic solution $\mathbf{u}_{a s}$ obtained at the limit when $t \rightarrow 0$ : The set $S$ consists of those boundary (or junction) points where $\mathbf{u}_{a s}$ does not obey the boundary conditions associated to operator $\mathcal{B}$, and of those interior points where $\mathbf{u}_{a s}$ is not smooth. In normal engineering situations $S$ is of measure zero, consisting typically of finitely many smooth open line segments (or closed smooth lines), such as the boundary line, and of isolated points, such as the points where concentrated loads are acting or the end points of the line segments in $S$.

Layers thus arise basically as 'line layers' or as 'hot spots' (point layers) and can be located by studying the (linear) asymptotics of the shell deformation in a given situation as $t \rightarrow$ 0 . This asymptotics is actually very case dependent, cf. [7, 24, 25], but we get the idea here by following the most often used asymptotic approach in the engineering shell theory, the so called membrane theory $[35,6]$. The mathematical shell-membrane theory is a linear 


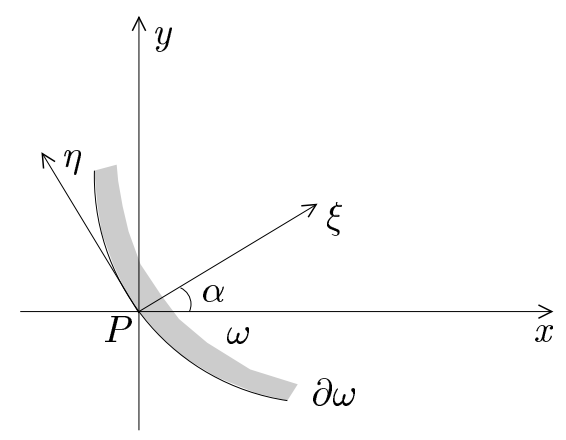

Figure 1: Boundary $(\xi, \eta)$ and principal curvature $(x, y)$ coordinates.

asymptotic theory obtained by setting $t=0$ in (1.1) and by carefully relaxing the boundary and interior regularity conditions on $\mathbf{u}$ accordingly, see [7, Part II] for the 'classicism' and $[30,31,32,22,20,21]$ for the 'modernism' on the subject.

Assume that $\omega$ is cut from a single smooth surface and has a piecewise smooth boundary line $\Gamma$. Assume also that the whole boundary is at least simply supported so that the displacements of the midsurface vanish along $\Gamma$. Then $S \supset \Gamma$, since the transverse deflection along $\Gamma$ cannot be restricted in the membrane theory [7, 22, 20, 21]. Furthermore, $S$ naturally contains the lines or points where the load $\mathbf{f}$ is irregular, say is discontinuous or involves concentrated line or point loads. Finally, in case of parabolic or hyperbolic shells (of zero or negative Gaussian curvature), $S$ may further contain characteristic lines along which the curvature vanishes. This is because, due to the hyperbolic nature of the reduced system (1.1) in the mentioned shell geometries, irregularities of $\mathbf{u}_{a s}$ (from various sources) propagate along characteristic lines $[22,21]$. At positive values of $t$, boundary or interior layers created by characteristic lines are particularly important since, as known from the classical shell theory [7, 28, 29] and also to be confirmed here, such layers have an exceptionally wide range. Often the amplitude is also strong so that such layers can dominate the whole deformation pattern, see $[9,10]$ for examples of benchmark problems of this kind.

In the shallow-shell models to be considered, Eqs. (1.1) take the form of a linear constantcoefficient system of PDE's when written in (approximate) principal curvature coordinates $x, y$ on $\omega$. The geometry of the shell is condensed in these models in three constant parameters: the principal radii of curvature $R_{1}, R_{2}$ (considered constant), and the thickness $t$. As a material parameter we introduce the Poisson ratio $\nu$ as usual (homogeneous isotropic material is assumed). Finally, the $\mathrm{RN}_{\mathrm{S}}$-model is parametrized additionally by a dimensionless shear (correction) factor to be denoted by $\gamma$. In this five-parameter setting, we study the nature of a 'line layer' generated by a single smooth line (segment) $S$. We make here a further geometric simplification by assuming that $S$ is 'shallow', i.e., smooth in length scale $\sim R$, so that we can treat $S$ approximately as a straight line when studying local effects in length scales $L \ll R$. The angle $\alpha$ formed by the assumed straight layer generator $S$ and the principal curvature line $y=0$ (Figure 1) is our sixth parameter. 
Along $S$ we assume finally a (finite or infinite) Fourier transform, so that we look for layer modes of the form

$$
\mathbf{u}_{l}^{k}(\xi, \eta)=e^{\lambda \xi} e^{i k \eta} \mathbf{U},
$$

where $\xi, \eta$ are rotated coordinates (Figure 1). Here the wave number $k$ is the seventh parameter in our study.

Once all the seven parameters above are fixed, the possible values of $\lambda$ in (1.2) are found as the roots of the characteristic polynomial of system (1.1), so that formally

$$
\lambda=\lambda\left(t ; k, R_{1}, R_{2}, \alpha, \nu, \gamma\right)
$$

Our mission is mainly to resolve this parametric dependence. We use Puiseux and Taylor expansions, taking $t$ as the primary parameter. The leading coefficients in the parametric expansions are evaluated by symbolic computation (MAPLE).

When a root $\lambda$ is found such that $\operatorname{Re} \lambda<0$, we may define the corresponding length scale of exponential decay (in the positive $\xi$-direction) as $L=-1 / \operatorname{Re} \lambda$. We are interested mainly in the 'layer roots' such that $L \rightarrow 0$ as $t \rightarrow 0$, but we will comment also on 'non-layers' ( $L$ independent of $t$ ) and 'anti-layers' $(L \rightarrow \infty$ as $t \rightarrow 0)$ arising in certain parametric regimes or in specific geometric situations. The plan, and the brief summary of the main results, is as follows.

In Section 2 we introduce the shallow shell models where our analysis is based upon. As the starting point we take the classical 2D model as presented in Novozhilov [19] (here named LNK). The shallow-shell approximations are made, leading to model $\mathrm{LNK}_{\mathrm{S}}$, and the $\mathrm{RN}_{\mathrm{S}}$-model is introduced as a parametrized variation of $\mathrm{LNK}_{\mathrm{S}}$. Finally the $\mathrm{LNK}_{\mathrm{SS}}$ - and $\mathrm{RN}_{\mathrm{SS}}$-models are introduced as an option for further simplification.

In Section 3 we find the layer solutions (1.2) to the homogeneous shell equations. We expand the characteristic roots here mainly as a function of $t$, holding the remaining six parameters fixed. We find that the layer scales vary with $t$ as $L \sim t^{1 / n}$ where $n \in\{1,2,3,4\}$, regardless of the shell model. Other exponents do not occur and the case $n=1$ appears in the $\mathrm{RN}_{\mathrm{S}}-$ model only. The layer mode with $n=1$ is found also in plates [1], so it is not related to curvature effects, but a consequence of shear deformation. Instead, the larger length scales, of which only one case can appear at a time, are the characteristic layer scales of a curved shell. The most common case $n=2$ is possible in all shell geometries. The other cases, where the layer has a still wider range, arise when the layer generating line $\xi=0$ is a characteristic line of the midsurface. This is possible in a hyperbolic shell $\left(R_{1} R_{2}<0\right)$, in which case $n=3$, or in a parabolic shell $\left(R_{1}=\infty,\left|R_{2}\right|<\infty\right)$, in which case $n=4$.

The mentioned main layer scales are known from the existing mathematical shell theory, see $[7,28,29]$. In the engineering theory of shells, the most thorough treatment of layers appears to be the one by Flügge [6]. In particular, Flügge analyzes the axial $(n=2)$ and angular $(n=4)$ layers in a cylindrical shell in a rather similar way as we do here. In [7], the case $n=2$ is characterized as a 'simple edge effect' and cases $n=3,4$ as 'generalized' edge effects. The different terminology here refers to the qualitatively different behaviour of the layer in variable $\eta$, as reflected in the parametric dependence on $k$ in our analysis. We find that the layer width 
varies with $k$ as $L \sim k^{-1+2 / n}$, so when $n=2$, there is no dependence on $k$, unlike in the other two cases. This means that in case $n=2$ the layer is a $1 \mathrm{D}$ effect in variable $\xi$ (parametrized by $\eta$ ), whereas in the other two cases, the layer arises as a non-local $2 \mathrm{D}$ phenomenon.

We complete the classical theory of shell layers here to a more systematic picture based on symbolic computation. The more detailed parametric analysis is carried out in Section 4, where we cover in particular the case when $k$ is variable and can be large (or small). We find that when $k$ exceeds the threshold $k \sim t^{-1 / 2}$, the curvature effects fade off and the deformation starts following the decoupled laws of a flat plate and membrane. In this regime, the characteristic shell layers degenerate to uniformly smooth 'non-layers' whose length scale $L$ is proportional to $k^{-1}$.

In Section 5 we finally add an 'energy perspective' to our results. Based on proper scaling of the coordinates and displacement components, as guided by our layer mode analysis, we find scaled deformation energies that are characteristic to shell layers. The energy expressions may be viewed as an extension of the asymptotic 'layer equations' found in the classical shell theory, cf. [7]. In the asymptotic analysis, the layer is viewed as a non-parametric smooth function in the scaled coordinates. Upon resolving the parametric dependence explicitly in the energy expressions, we can further show how the layer is effectively seen by the finite element solver that attempts to resolve the layer by minimizing energy. Our results should be useful in the further analysis of numerical locking effects (and their possible remedies) when it comes to FEM approximation of shell layers.

\section{The shallow shell models}

We start from the classical 2D shell model as formulated in Novozhilov [19]. Assume that the midsurface $\omega$ lies on a given smooth surface parametrized globally by curvilinear coordinates $\alpha_{1}, \alpha_{2}$. As in [19] we assume principal curvature coordinates, so that only four scalar parameters are needed to specify the curvature and the metric on $\omega$. These are the principal curvatures, here denoted by $b_{1}, b_{2}$, and the metric parameters $A_{1}, A_{2}$ relating coordinate changes to arc lengths. Following [19] we write the displacement vector field of the midsurface then as

$$
\vec{u}=u \vec{e}_{1}+v \vec{e}_{2}+w \vec{e}_{3},
$$

where $\vec{e}_{1}, \vec{e}_{2}$ are the unit tangent vectors along the principal curvature lines and $\vec{e}_{3}$ is the unit normal.

In the assumed (classical, cf. also [14]) notation one has $\mathbf{u}=(u, v, w)$ in Eq. (1.1). When $\mathbf{f}=\mathbf{0}$, this system arises as the Euler equation of the principle of minimum strain energy. The scaled expression of the latter, in case of homogeneous isotropic material with Poisson ratio $\nu$, is given by [19, Eq. (9.12)]

$$
\begin{aligned}
\mathcal{F}(\mathbf{u}) & =\frac{1}{2} \int_{\omega}\left[\nu\left(\beta_{11}+\beta_{22}\right)^{2}+(1-\nu)\left(\beta_{11}^{2}+2 \beta_{12}^{2}+\beta_{22}^{2}\right)\right] A_{1} A_{2} d \alpha_{1} d \alpha_{2} \\
& +\frac{t^{2}}{24} \int_{\omega}\left[\nu\left(\kappa_{11}+\kappa_{22}\right)^{2}+(1-\nu)\left(\kappa_{11}^{2}+2 \kappa_{12}^{2}+\kappa_{22}^{2}\right)\right] A_{1} A_{2} d \alpha_{1} d \alpha_{2}
\end{aligned}
$$


where $\beta_{i j}$ and $\kappa_{i j}$ denote the components of the membrane strain (related to change of metric) and bending strain (related to change of curvature) tensors, respectively. These are defined as [19, Eq. (4.23)]

$$
\begin{aligned}
& \beta_{11}=\frac{1}{A_{1}} \frac{\partial u}{\partial \alpha_{1}}+\frac{1}{A_{1} A_{2}} \frac{\partial A_{1}}{\partial \alpha_{2}} v+b_{1} w \\
& \beta_{22}=\frac{1}{A_{2}} \frac{\partial v}{\partial \alpha_{2}}+\frac{1}{A_{1} A_{2}} \frac{\partial A_{2}}{\partial \alpha_{1}} u+b_{2} w \\
& \beta_{12}=\frac{A_{1}}{2 A_{2}} \frac{\partial}{\partial \alpha_{2}}\left(\frac{u}{A_{1}}\right)+\frac{A_{2}}{2 A_{1}} \frac{\partial}{\partial \alpha_{1}}\left(\frac{v}{A_{2}}\right)
\end{aligned}
$$

and

$$
\begin{aligned}
\kappa_{11} & =\frac{1}{A_{1}} \frac{\partial}{\partial \alpha_{1}}\left(\frac{1}{A_{1}} \frac{\partial w}{\partial \alpha_{1}}-b_{1} u\right)+\frac{1}{A_{1} A_{2}} \frac{\partial A_{1}}{\partial \alpha_{2}}\left(\frac{1}{A_{2}} \frac{\partial w}{\partial \alpha_{2}}-b_{2} v\right), \\
\kappa_{22} & =\frac{1}{A_{2}} \frac{\partial}{\partial \alpha_{2}}\left(\frac{1}{A_{2}} \frac{\partial w}{\partial \alpha_{2}}-b_{2} v\right)+\frac{1}{A_{1} A_{2}} \frac{\partial A_{2}}{\partial \alpha_{1}}\left(\frac{1}{A_{1}} \frac{\partial w}{\partial \alpha_{1}}-b_{1} u\right), \\
\kappa_{12} & =\frac{1}{A_{1} A_{2}}\left(\frac{\partial^{2} w}{\partial \alpha_{1} \partial \alpha_{2}}-\frac{1}{A_{1}} \frac{\partial A_{1}}{\partial \alpha_{2}} \frac{\partial w}{\partial \alpha_{1}}-\frac{1}{A_{2}} \frac{\partial A_{2}}{\partial \alpha_{1}} \frac{\partial w}{\partial \alpha_{2}}\right) \\
& -b_{1}\left(\frac{1}{A_{2}} \frac{\partial u}{\partial \alpha_{2}}-\frac{1}{A_{1} A_{2}} \frac{\partial A_{1}}{\partial \alpha_{2}} u\right)-b_{2}\left(\frac{1}{A_{1}} \frac{\partial v}{\partial \alpha_{1}}-\frac{1}{A_{1} A_{2}} \frac{\partial A_{2}}{\partial \alpha_{1}} v\right) .
\end{aligned}
$$

Here we have assumed that $b_{i}(P)>0$ when the corresponding center of curvature lies in the negative normal direction from $P$.

Let us note in this context that, while the expressions (2.2) of the membrane strains were stated already by Love in his pioneering work [13, 14], the bending strains have been the subject of a long discussion, and a number of slightly different expressions have been proposed over the years. For example, Love's (later) proposal [14] relates to Novozhilov's as

$$
\kappa_{11}^{L}=\kappa_{11}^{N}, \quad \kappa_{22}^{L}=\kappa_{22}^{N}, \quad \kappa_{12}^{L}=\kappa_{12}^{N}+2 b_{1} \beta_{12},
$$

whereas Vlasov [35] suggests

$$
\kappa_{11}^{V}=\kappa_{11}^{N}+b_{1} \beta_{11}, \quad \kappa_{22}^{V}=\kappa_{22}^{N}+b_{2} \beta_{22}, \quad \kappa_{12}^{V}=\kappa_{12}^{N}+\left(b_{1}+b_{2}\right) \beta_{12} .
$$

(Here we have used the Codazzi relations [19, Eq.(2.28)] as well.) The final word in this discussion, usually referred to as the Koiter model [11, 12], is a consistent first-approximation model derived from the exact linearized change of metric and change of curvature tensors in general coordinates, cf. [4]. In the present notation, the Koiter bending strains relate to Novozhilov's as

$$
\kappa_{11}^{K}=\kappa_{11}^{N}+b_{1} \beta_{11}, \quad \kappa_{22}^{K}=\kappa_{22}^{N}+b_{2} \beta_{22}, \quad \kappa_{12}^{K}=\kappa_{12}^{N} .
$$

Below we choose to work with Novozhilov's expressions (2.3) as these appear the simplest ones for our purposes. - Note that in view of (2.1), any modification of $\kappa_{i j}$ by an added linear 
combination of $\beta_{i j}$ 's only causes a small regular perturbation of the energy when $t$ is small. In our study such model variations would be irrelevant. We name the chosen model (2.1)-(2.3) here as LNK according to Love-Novozhilov-Koiter.

We introduce next the shallow shell approximations within the LNK-model. For a given point $P \in \omega$, let us scale the coordinates $\alpha_{1}, \alpha_{2}$ to be the arc lengths from $P$ along the coordinate lines, so that $P=(0,0)$ and $A_{1}\left(\alpha_{1}, 0\right)=A_{2}\left(0, \alpha_{2}\right)=1$. Our aim is to simplify the expressions $(2.2)-(2.3)$ by approximate expansions around $P$.

To begin with, let us introduce the localized geometric parameters

$$
\begin{aligned}
\frac{1}{R_{1}} & =b_{1}(P), \quad \frac{1}{R_{2}}=b_{2}(P), \quad c_{i j}=\frac{\partial b_{i}}{\partial \alpha_{j}}(P), \quad i, j=1,2, \\
a_{1} & =\frac{\partial A_{1}}{\partial \alpha_{2}}(P), \quad a_{2}=\frac{\partial A_{2}}{\partial \alpha_{1}}(P) .
\end{aligned}
$$

These are related by the Codazzi relations [19, Eq.(2.28)] as

$$
\left(\frac{1}{R_{j}}-\frac{1}{R_{i}}\right) a_{i}=c_{i j} \quad \text { when } \quad i \neq j
$$

We will measure the 'total curvature' of the shell by $R$, defined by

$$
\frac{1}{R}=\left(\frac{1}{R_{1}^{2}}+\frac{1}{R_{2}^{2}}\right)^{1 / 2}
$$

We assume that $R$ is finite and that the surface containing $\omega$ varies smoothly in the length scale $R$. We may then call $R$ the curvature length scale on $\omega$.

The first step in the derivation of the shallow shell model is to rectify the metric at $P$ by passing to new coordinates $x, y$, normally thought of as the rectilinear coordinates on the tangent plane to $\omega$ at $P$. Here we choose quadratic approximations of such coordinates along $\omega$, as given by

$$
x=\alpha_{1}+a_{1} \alpha_{1} \alpha_{2}-\frac{a_{2}}{2} \alpha_{2}^{2}, \quad y=\alpha_{2}+a_{2} \alpha_{1} \alpha_{2}-\frac{a_{1}}{2} \alpha_{1}^{2},
$$

where $a_{1}, a_{2}$ are defined by (2.4). This transform is obviously one-to-one at least locally around $P$.

Passing now to the new coordinates $x, y$ we have

$$
A_{1}(x, y)=1+a_{1} y+\cdots, \quad A_{2}(x, y)=1+a_{2} x+\cdots,
$$

and

$$
\begin{aligned}
\frac{\partial}{\partial \alpha_{1}} & =\left(1+a_{1} y+\cdots\right) \frac{\partial}{\partial x}+\left(a_{2} y-a_{1} x+\cdots\right) \frac{\partial}{\partial y} \\
\frac{\partial}{\partial \alpha_{2}} & =\left(a_{1} x-a_{2} y+\cdots\right) \frac{\partial}{\partial x}+\left(1+a_{2} x+\cdots\right) \frac{\partial}{\partial y}
\end{aligned}
$$


where the dropped terms are of relative order $O\left((x / R)^{2}+(y / R)^{2}\right)$. Likewise, writing

$$
u \vec{e}_{1}+v \vec{e}_{2}=\tilde{u} \vec{e}_{x}+\tilde{v} \vec{e}_{y},
$$

where $\vec{e}_{x}, \vec{e}_{y}$ are the unit tangent vectors along the new coordinate lines on $\omega$, we have

$$
u=\tilde{u}+\left(a_{2} y-a_{1} x\right) \tilde{v}+\cdots, \quad v=\tilde{v}+\left(a_{1} x-a_{2} y\right) \tilde{u}+\cdots .
$$

The second step in the shallow shell approximation is to deflect the third displacement component $w$ slightly from the normal direction, by defining $\tilde{w}$ so that

$$
w=\tilde{w}+\left(\frac{1}{2} c_{11} x^{2}+\frac{1}{2} c_{21} y^{2}+c_{12} x y\right) \tilde{u}+\left(\frac{1}{2} c_{12} x^{2}+\frac{1}{2} c_{22} y^{2}+c_{21} x y\right) \tilde{v} .
$$

Using now (2.8)-(2.10), we can expand the strains (2.2)-(2.3) around $P$ as

$$
\beta_{11}=\frac{\partial \tilde{u}}{\partial x}+\frac{\tilde{w}}{R_{1}}+\cdots, \quad \beta_{22}=\frac{\partial \tilde{v}}{\partial y}+\frac{\tilde{w}}{R_{2}}+\cdots, \quad \beta_{12}=\frac{1}{2}\left(\frac{\partial \tilde{u}}{\partial y}+\frac{\partial \tilde{v}}{\partial x}\right)+\cdots
$$

and, using also (2.5),

$$
\begin{aligned}
& \kappa_{11}=\frac{\partial^{2} \tilde{w}}{\partial x^{2}}-\frac{1}{R_{1}} \frac{\partial \tilde{u}}{\partial x}+\cdots, \quad \kappa_{22}=\frac{\partial^{2} \tilde{w}}{\partial y^{2}}-\frac{1}{R_{2}} \frac{\partial \tilde{v}}{\partial y}+\cdots, \\
& \kappa_{12}=\frac{\partial^{2} \tilde{w}}{\partial x \partial y}-\frac{1}{R_{1}} \frac{\partial \tilde{u}}{\partial y}-\frac{1}{R_{2}} \frac{\partial \tilde{v}}{\partial x}+\cdots .
\end{aligned}
$$

Here the coefficients of the neglected terms are of relative order $O(|x| / R+|y| / R)$. Up to this accuracy, we may also set

$$
A_{1} A_{2} d \alpha_{1} d \alpha_{2} \hookrightarrow d x d y
$$

when evaluating the strain energy (2.1).

Our shallow approximation within the LNK-model is now simply to use (2.13) in the energy expression (2.1) and to define the strains there by truncating the expansions (2.11)-(2.12) to the terms shown. Dropping henceforth the tildes, we assume thus the simplifies strain expressions

$$
\beta_{11}=\frac{\partial u}{\partial x}+\frac{w}{R_{1}}, \quad \beta_{22}=\frac{\partial v}{\partial y}+\frac{w}{R_{2}}, \quad \beta_{12}=\frac{1}{2}\left(\frac{\partial u}{\partial y}+\frac{\partial v}{\partial x}\right),
$$

and

$$
\begin{aligned}
& \kappa_{11}=\frac{\partial^{2} w}{\partial x^{2}}-\frac{1}{R_{1}} \frac{\partial u}{\partial x}, \quad \kappa_{22}=\frac{\partial^{2} w}{\partial y^{2}}-\frac{1}{R_{2}} \frac{\partial v}{\partial y}, \\
& \kappa_{12}=\frac{\partial^{2} w}{\partial x \partial y}-\frac{1}{R_{1}} \frac{\partial u}{\partial y}-\frac{1}{R_{2}} \frac{\partial v}{\partial x}
\end{aligned}
$$

where now the localized principal curvatures $R_{i}^{-1}$ are the only geometric parameters. We name the model $(2.1) \&(2.13) \&(2.14) \&(2.15)$ as $\mathrm{LNK}_{\mathrm{S}}$. This is the basic model in our study. 
Remark 2.1 When $\omega$ varies smoothly in the curvature length scale $R$, as assumed, the error of the above approximation is formally of order $O(L / R)$ where $L$ is the characteristic dimension of $\omega$. The $\mathrm{LNK}_{\mathrm{S}}$-model may then be viewed as the asymptotic model corresponding to the parametric limit $\epsilon=L / R \rightarrow 0$. Note also that under the assumptions made, the deviation of $\omega$ from its tangent plane at $P$ is at most of order $d \sim L^{2} / R=\epsilon^{2} R$, so when $\epsilon$ is small, the shell is indeed shallow. We should underline that this concept is not related to the thickness parameter $t$ at all.

Remark 2.2 In view of (2.10), the dropped terms in (2.12) involve second derivatives of $\tilde{u}, \tilde{v}$, so the truncation is somewhat 'singular' here. This is avoided in the special case where $c_{i j}=0$, i.e., when the curvatures are slowly varying at $P$. One has then also $a_{i}=0$ by (2.5) (this holds also when $\left.R_{1}=R_{2}\right)$, so in fact then $(\tilde{u}, \tilde{v}, \tilde{w})=(u, v, w)$ above. In the even more special case of a cylindrical shell, where $R_{1}=\infty, R_{2}=R$ is constant, and $A_{1}=A_{2}=1$ throughout $\omega$, we actually end up making no approximation above, so $\mathrm{LNK}=\mathrm{LNK}_{\mathrm{S}}$ in that case. Then $\mathrm{LNK}_{\mathrm{S}}$ may be considered an approximate model even for non-shallow shell geometries that are small variations from the cylindrical shape, i.e., axially shallow.

When not aiming at a model valid for a cylindrical shell, we may derive a simpler shallow shell model by assuming instead of (2.10) the transform

$$
\begin{aligned}
w=\left(1+\frac{x^{2}}{2 R_{1}^{2}}+\frac{y^{2}}{2 R_{2}^{2}}\right) \tilde{w} & +\left(\frac{x}{R_{1}}+\frac{1}{2} c_{11} x^{2}+\frac{1}{2} c_{21} y^{2}+c_{12} x y\right) \tilde{u} \\
& +\left(\frac{y}{R_{2}}+\frac{1}{2} c_{12} x^{2}+\frac{1}{2} c_{22} y^{2}+c_{21} x y\right) \tilde{v}
\end{aligned}
$$

for defining $\tilde{w}$. Note that here $\tilde{w}$ is defined essentially as the quadratic approximation of the displacement component in the direction $\vec{e}_{z}=\vec{e}_{3}(P)$. When using (2.9), (2.16), the membrane strain expansions (2.11) remain valid, but the bending strains are now expanded as

$$
\begin{aligned}
\kappa_{11} & =\frac{\partial^{2} \tilde{w}}{\partial x^{2}}+\frac{1}{R_{1}}\left(\frac{\partial \tilde{u}}{\partial x}+\frac{\tilde{w}}{R_{1}}\right)+\cdots, \quad \kappa_{22}=\frac{\partial^{2} \tilde{w}}{\partial y^{2}}+\frac{1}{R_{2}}\left(\frac{\partial \tilde{v}}{\partial y}+\frac{\tilde{w}}{R_{2}}\right)+\cdots, \\
\kappa_{12} & =\frac{\partial^{2} \tilde{w}}{\partial x \partial y}+\cdots
\end{aligned}
$$

Neglecting here the second terms in $\kappa_{i i}$ (as these have little effect in the energy, see the remarks above on different shell models), and dropping the tildes we end up simplifying the bending strains as

$$
\kappa_{11}=\frac{\partial^{2} w}{\partial x^{2}}, \quad \kappa_{22}=\frac{\partial^{2} w}{\partial y^{2}}, \quad \kappa_{12}=\frac{\partial^{2} w}{\partial x \partial y},
$$

as corresponding to the Kirchhoff model of plate bending. Below we refer to the model (2.1) $\&(2.13) \&(2.14) \&(2.17)$ as $\mathrm{LNK}_{\mathrm{SS}}$.

We introduce finally a variant of the $\mathrm{LNK}_{\mathrm{S}}$ shell model where the so called rotations $\theta, \psi$ are added to the displacement field, so as to make $\mathbf{u}$ a five-component field. In the shallow 
version of this model, the strain energy is written as

$$
\begin{aligned}
\mathcal{F}(\mathbf{u}) & =\frac{1}{2} \int_{\omega}\left[\nu\left(\beta_{11}+\beta_{22}\right)^{2}+(1-\nu)\left(\beta_{11}^{2}+2 \beta_{12}^{2}+\beta_{22}^{2}\right)\right] d x d y \\
& +\frac{\gamma}{4}(1-\nu) \int_{\omega}\left(\rho_{1}^{2}+\rho_{2}^{2}\right) d x d y \\
& +\frac{t^{2}}{24} \int_{\omega}\left[\nu\left(\kappa_{11}+\kappa_{22}\right)^{2}+(1-\nu)\left(\kappa_{11}^{2}+2 \kappa_{12}^{2}+\kappa_{22}^{2}\right)\right] d x d y
\end{aligned}
$$

where the second term arises from the assumed transverse shear deformations. We consider here $\gamma$ (the so called shear factor) as a free parameter $(\gamma>0)$. In $(2.18)$, the membrane strains are again defined by (2.14), the transverse shear strains $\rho_{1}, \rho_{2}$ are given by

$$
\rho_{1}=\theta-\frac{\partial w}{\partial x}+\frac{u}{R_{1}}, \quad \rho_{2}=\psi-\frac{\partial w}{\partial y}+\frac{v}{R_{2}},
$$

and the bending strains by

$$
\begin{aligned}
& \kappa_{11}=\frac{\partial \theta}{\partial x}, \quad \kappa_{22}=\frac{\partial \psi}{\partial y} \\
& \kappa_{12}=\frac{1}{2}\left(\frac{\partial \theta}{\partial y}+\frac{\partial \psi}{\partial x}-\frac{1}{R_{1}} \frac{\partial u}{\partial y}-\frac{1}{R_{2}} \frac{\partial v}{\partial x}\right) .
\end{aligned}
$$

Model (2.18) \& (2.14) \& (2.19) \& (2.20) has its engineering roots in plate (or beam) theories, due to Reissner [26] and others. For non-shallow shells in general coordinates, a consistent derivation in the Koiter spirit was given by Naghdi $[16,17]$. We name the model $(2.18)$ \& (2.14) \& (2.19) \& (2.20) here as $\mathrm{RN}_{\mathrm{S}}$ according to Reissner-Naghdi. Note that $\mathrm{RN}_{\mathrm{S}}$ with $\gamma=\infty$ gives $\mathrm{LNK}_{\mathrm{S}}$, so $\mathrm{RN}_{\mathrm{S}}$ may be viewed as a parametric extension of $\mathrm{LNK}_{\mathrm{S}}$.

When the curvature dependent terms in (2.19)-(2.20) are dropped, one obtains a variant of the $\mathrm{LNK}_{\mathrm{SS}}$-model where the transverse shear and bending strains are defined by

$$
\begin{aligned}
\rho_{1} & =\theta-\frac{\partial w}{\partial x}, \quad \rho_{2}=\psi-\frac{\partial w}{\partial y}, \\
\kappa_{11} & =\frac{\partial \theta}{\partial x}, \quad \kappa_{22}=\frac{\partial \psi}{\partial y}, \quad \kappa_{12}=\frac{1}{2}\left(\frac{\partial \theta}{\partial y}+\frac{\partial \psi}{\partial x}\right),
\end{aligned}
$$

as corresponding to the Reissner-Mindlin model for plate bending. The model (2.18) \& (2.14) $\&(2.21)$ is named $\mathrm{RN}_{\mathrm{SS}}$.

\section{Zero thickness asymptotics of shell layers}

The boundary layers are non-trivial solutions of the homogeneous Euler-Lagrange equations corresponding to (1.1). We consider here the dimensionally reduced 2-D shallow shell models without shear $\mathrm{LNK}_{\mathrm{S}}$ (i.e., of Koiter type), and with shear $\mathrm{RN}_{\mathrm{S}}$ (i.e., of Naghdi type) in the approximate principal curvature coordinates $x, y$ as chosen above. The Euler-Lagrange equations can be written for the $\mathrm{LNK}_{\mathrm{S}}$ model as 


$$
\begin{aligned}
0 & =-\frac{\partial \beta_{11}}{\partial x}-\nu \frac{\partial \beta_{22}}{\partial x}-(1-\nu) \frac{\partial \beta_{12}}{\partial y}+\frac{t^{2}}{12 R_{1}}\left(\frac{\partial \kappa_{11}}{\partial x}+\nu \frac{\partial \kappa_{22}}{\partial x}+2(1-\nu) \frac{\partial \kappa_{12}}{\partial y}\right), \\
0 & =-\nu \frac{\partial \beta_{11}}{\partial y}-\frac{\partial \beta_{22}}{\partial y}-(1-\nu) \frac{\partial \beta_{12}}{\partial x}+\frac{t^{2}}{12 R_{2}}\left(\nu \frac{\partial \kappa_{11}}{\partial y}+\frac{\partial \kappa_{22}}{\partial y}+2(1-\nu) \frac{\partial \kappa_{12}}{\partial x}\right), \\
0 & \left(\frac{1}{R_{1}}+\frac{\nu}{R_{2}}\right) \beta_{11}+\left(\frac{\nu}{R_{1}}+\frac{1}{R_{2}}\right) \beta_{22} \\
& +\frac{t^{2}}{12}\left(\frac{\partial^{2} \kappa_{11}}{\partial^{2} x}+\nu \frac{\partial^{2} \kappa_{11}}{\partial^{2} y}+\nu \frac{\partial^{2} \kappa_{22}}{\partial^{2} x}+\frac{\partial^{2} \kappa_{22}}{\partial^{2} y}+2(1-\nu) \frac{\partial^{2} \kappa_{12}}{\partial x \partial y}\right),
\end{aligned}
$$

and for the $\mathrm{RN}_{\mathrm{S}}$ model as

$$
\begin{aligned}
0 & =-\frac{\partial \beta_{11}}{\partial x}-\nu \frac{\partial \beta_{22}}{\partial x}-(1-\nu) \frac{\partial \beta_{12}}{\partial y}+\frac{1-\nu}{2} \gamma \frac{\rho_{1}}{R_{1}}+\frac{t^{2}}{12}(1-\nu) \frac{1}{R_{1}} \frac{\partial \kappa_{12}}{\partial y}, \\
0 & =-\nu \frac{\partial \beta_{11}}{\partial y}-\frac{\partial \beta_{22}}{\partial y}-(1-\nu) \frac{\partial \beta_{12}}{\partial x}+\frac{1-\nu}{2} \gamma \frac{\rho_{2}}{R_{2}}+\frac{t^{2}}{12}(1-\nu) \frac{1}{R_{2}} \frac{\partial \kappa_{12}}{\partial x} \\
0 & =\left(\frac{1}{R_{1}}+\frac{\nu}{R_{2}}\right) \beta_{11}+\left(\frac{\nu}{R_{1}}+\frac{1}{R_{2}}\right) \beta_{22}+\frac{1-\nu}{2} \gamma\left(\frac{\partial \rho_{1}}{\partial x}+\frac{\partial \rho_{2}}{\partial y}\right) \\
0 & =\frac{1-\nu}{2} \gamma \rho_{1}-\frac{t^{2}}{12}\left(\frac{\partial \kappa_{11}}{\partial x}+\nu \frac{\partial \kappa_{22}}{\partial x}+(1-\nu) \frac{\partial \kappa_{12}}{\partial y}\right) \\
0 & =\frac{1-\nu}{2} \gamma \rho_{2}-\frac{t^{2}}{12}\left(\nu \frac{\partial \kappa_{11}}{\partial y}+\frac{\partial \kappa_{22}}{\partial y}+(1-\nu) \frac{\partial \kappa_{12}}{\partial x}\right) .
\end{aligned}
$$

Here the strain-displacement relations are given by (2.14)-(2.15) for the $\mathrm{LNK}_{\mathrm{S}}$ model and by (2.14), (2.19)-(2.20) for the $\mathrm{RN}_{\mathrm{S}}$ model.

\subsection{The main boundary layer modes}

The boundary layer analysis is based on assuming in (3.1) and (3.2) the Ansatz (1.2) in the rotated coordinates $\xi=\cos \alpha x+\sin \alpha y, \eta=-\sin \alpha x+\cos \alpha y$ (see Figure 1). We start from the $\mathrm{LNK}_{\mathrm{S}}$ model. In this case, substituting (1.2) and

$$
\frac{\partial}{\partial x}=\cos \alpha \frac{\partial}{\partial \xi}-\sin \alpha \frac{\partial}{\partial \eta}, \quad \frac{\partial}{\partial y}=\sin \alpha \frac{\partial}{\partial \xi}+\cos \alpha \frac{\partial}{\partial \eta}
$$

in $(3.1) \&(2.14)-(2.15)$, we obtain the characteristic equation

$$
P_{\mathrm{LNK}_{\mathrm{S}}}\left(\lambda ; t, k, \nu, \alpha, R_{1}, R_{2}\right)=0,
$$

where $P_{\mathrm{LNK}_{\mathrm{S}}}$ is a polynomial in $\lambda$ and $t$ and can be expanded as 


$$
\begin{aligned}
P_{\mathrm{LNK}_{\mathrm{S}}}\left(\lambda ; t, k, \nu, \alpha, R_{1}, R_{2}\right) & =t^{2} a_{8}(t) \lambda^{8}+t^{4} a_{7}(t) \lambda^{7}+t^{2} a_{6}(t) \lambda^{6}+t^{2} a_{5}(t) \lambda^{5} \\
& +a_{4}(t) \lambda^{4}+a_{3}(t) \lambda^{3}+a_{2}(t) \lambda^{2}+a_{1}(t) \lambda+a_{0}(t),
\end{aligned}
$$

where

$$
\begin{array}{ll}
a_{8}(t)=a_{80}+a_{82} t^{2}+a_{84} t^{4}, & a_{7}(t)=a_{70}+a_{72} t^{2}, \\
a_{6}(t)=a_{60}+a_{62} t^{2}+a_{64} t^{4}, & a_{5}(t)=a_{50}+a_{52} t^{2}+a_{54} t^{4}, \\
a_{4}(t)=a_{40}+a_{42} t^{2}+a_{44} t^{4}+a_{46} t^{6}, & a_{3}(t)=a_{30}+a_{32} t^{2}+a_{34} t^{4}+a_{36} t^{6}, \\
a_{2}(t)=a_{20}+a_{22} t^{2}+a_{24} t^{4}+a_{26} t^{6}, & a_{1}(t)=a_{10}+a_{12} t^{2}+a_{14} t^{4}+a_{16} t^{6}, \\
a_{0}(t)=a_{00}+a_{02} t^{2}+a_{04} t^{4}+a_{06} t^{6} . &
\end{array}
$$

The boundary layer length scales for the shallow shell are determined by the asymptotic behaviour of the roots $\lambda(t)$ of the characteristic equation (3.3) as $t \rightarrow 0$ : a family $\{\lambda(t)\}_{t}$ of roots with $|\lambda(t)| \rightarrow \infty, \operatorname{Re} \lambda(t)<0$, as $t \rightarrow 0$ corresponds to true layer solutions. We will also find solutions with $|\lambda(t)| \rightarrow 0$, as corresponding to 'anti-layer' modes that degenerate to polynomials in $\xi$ at $t=0$ (with the degree determined by the multiplicity of $\lambda(0)=0$ ). Below we present the asymptotic analysis of these eigenvalues in dependence of $t$ while keeping the other parameters fixed.

The following leading coefficients in (3.5) turn out to be decisive for the main boundary layer effects:

$$
\begin{aligned}
& a_{80}=\frac{1}{24}(1-\nu), \\
& a_{40}=\frac{1}{2}\left(1-\nu^{2}\right)(1-\nu)\left(\frac{\sin ^{2} \alpha}{R_{1}}+\frac{\cos ^{2} \alpha}{R_{2}}\right)^{2}, \\
& a_{30}=2 i k\left(1-\nu^{2}\right)(1-\nu)\left(\frac{1}{R_{1}}-\frac{1}{R_{2}}\right)\left(\frac{\sin ^{2} \alpha}{R_{1}}+\frac{\cos ^{2} \alpha}{R_{2}}\right) \sin \alpha \cos \alpha, \\
& a_{20}=-\left(1-\nu^{2}\right)(1-\nu) k^{2}\left(3\left(\frac{1}{R_{1}}-\frac{1}{R_{2}}\right)^{2} \sin ^{2} \alpha \cos ^{2} \alpha+\frac{1}{R_{1} R_{2}}\right), \\
& a_{10}=-2 i k^{3}\left(1-\nu^{2}\right)(1-\nu)\left(\frac{1}{R_{1}}-\frac{1}{R_{2}}\right)\left(\frac{\cos ^{2} \alpha}{R_{1}}+\frac{\sin ^{2} \alpha}{R_{2}}\right) \sin \alpha \cos \alpha, \\
& a_{00}=\frac{1}{2}\left(1-\nu^{2}\right)(1-\nu) k^{4}\left(\frac{\cos ^{2} \alpha}{R_{1}}+\frac{\sin ^{2} \alpha}{R_{2}}\right)^{2} \cdot
\end{aligned}
$$

We see that these coefficients are in turn depending on the following geometric quantities (which may be interpreted as mid-surface curvatures along the $\eta$-axis, respectively $\xi$-axis):

$$
a_{\eta}=\frac{\sin ^{2} \alpha}{R_{1}}+\frac{\cos ^{2} \alpha}{R_{2}}, \quad a_{\xi}=\frac{\cos ^{2} \alpha}{R_{1}}+\frac{\sin ^{2} \alpha}{R_{2}} .
$$


The aim of this section is to provide a complete analysis of the dependence of the eigenvalues $\lambda_{i}, i=1, \ldots, 8$, with respect to the shell parameters, with emphasis on the dependence with respect to the thickness of the shell $t$. We shall see below that $\lambda_{i}$ can be expanded as Puiseux series of $t$, in a sufficiently small neighbourhood of 0 . Of special interest is also the leading term of these expansions, $\mu_{0, t^{\beta}}$, where

$$
\lambda=\lambda(t)=t^{-\beta} z\left(t^{\beta}\right)
$$

and $z=z(s)=\sum_{j \geq 0} \mu_{j, t^{\beta}} s^{j}$ is an analytic function in a small neighbourhood of 0 .

We shall also see that $\beta$ can take only the values $1 / 4,1 / 3,1 / 2$, i.e., we can only get the length scales $L \sim \sqrt[4]{t}, \sqrt[3]{t}, \sqrt{t}$, and we make a careful analysis of the cases with respect to the local geometry of the shell.

\subsubsection{The basic edge effect}

Let us first investigate what conditions should the coefficients $a_{i j}$ satisfy, such that we get $\sqrt{t}$ length scale eigenvalues. Assume that $\lambda=\lambda(t)=z(\sqrt{t}) / \sqrt{t}$ is such a solution of the characteristic equation (3.3), with $z(\cdot)$ analytic function in a small neighbourhood of 0 .

Substituting this into the characteristic equation, together with $s=\sqrt{t}$, and multiplying then with $s^{4}$, we find that $z$ should be solution of the following equation

$$
\begin{aligned}
Q^{\sqrt{t}}(z, s) & :=a_{8}\left(s^{2}\right) z^{8}+s^{5} a_{7}\left(s^{2}\right) z^{7}+s^{2} a_{6}\left(s^{2}\right) z^{6}+s^{3} a_{5}\left(s^{2}\right) z^{5} \\
& +a_{4}\left(s^{2}\right) z^{4}+s a_{3}\left(s^{2}\right) z^{3}+s^{2} a_{2}\left(s^{2}\right) z^{2}+s^{3} a_{1}\left(s^{2}\right) z+s^{4} a_{0}\left(s^{2}\right)=0 .
\end{aligned}
$$

Taking the limit $s \rightarrow 0$ in (3.8), we find that the leading term $\mu_{0, \sqrt{t}}$ of the series expansion of $z(s)=\sum_{j=0}^{\infty} \mu_{j, \sqrt{t}} s^{j}$ satisfies the following equation:

$$
\left(a_{80}\left(\mu_{0, \sqrt{t}}\right)^{4}+a_{40}\right)\left(\mu_{0, \sqrt{t}}\right)^{4}=0 .
$$

Proposition 3.1 Assume that at the boundary point $(x, y)=(0,0)$ the curvature along $\eta$-axis does not vanish, i.e., $a_{\eta} \neq 0$. Then four of the eight roots of the characteristic polynomial $P_{L N K_{S}}$ in (3.4) correspond to a boundary layer of thickness $O(\sqrt{t})$. These roots take the form $\lambda(t)=z(\sqrt{t}) / \sqrt{t}$, where $z(s)=\sum_{j=0}^{\infty} \mu_{j, \sqrt{t}} s^{j}$ is an analytic function in a small neighbourhood of 0 , and the leading term of the expansion is $\mu_{0, \sqrt{t}}=\rho\left(12\left(1-\nu^{2}\right) a_{\eta}^{2}\right)^{1 / 4}$, with $\rho^{4}+1=0$.

The remaining 4 roots correspond to 'long range' effects and have the form $\lambda(t)=\sum_{j=0}^{\infty} c_{j, \sqrt{t}} t^{j / 2}$, where

$$
c_{0, \sqrt{t}}=\frac{k}{a_{\eta}}\left[-i\left(\frac{1}{R_{1}}-\frac{1}{R_{2}}\right) \sin \alpha \cos \alpha \pm \frac{1}{\sqrt{R_{1} R_{2}}}\right] .
$$

Proof. Since $\mu_{0, \sqrt{t}}$ solves the equation (3.9) we get nontrivial solutions only if $a_{40} \neq 0$. The hypothesis $a_{\eta} \neq 0$ implies that for the equation (3.9) we get 4 trivial and 4 nontrivial solutions. 
The formula of the leading term follows then immediately, since from $(3.6 b)$ and $(3.6 a)$ we see that

$$
a_{40}=\frac{1}{2}\left(1-\nu^{2}\right)(1-\nu) a_{\eta}^{2}, \quad a_{80}=\frac{1}{24}(1-\nu)
$$

This implies that

$$
\mu_{0, \sqrt{t}}=\rho \sqrt[4]{\frac{a_{40}}{a_{80}}}
$$

with $\rho^{4}+1=0$. Let us denote by $\mu_{0, \sqrt{t}}^{l}, l=1, \ldots, 4$, the four nontrivial solutions of equation (3.9). Therefore, since

$$
Q^{\sqrt{t}}\left(\mu_{0, \sqrt{t}}^{l}, 0\right)=0, \quad \frac{\partial Q^{\sqrt{t}}}{\partial z}\left(\mu_{0, \sqrt{t}}^{l}, 0\right) \neq 0, \quad l=1, \ldots, 4,
$$

and $Q^{\sqrt{t}}(\cdot, \cdot)$ is an analytic function of both variables, the Implicit Function Theorem implies that there exist $\varepsilon>0$ and $z^{l}(\cdot) \in \mathcal{A}(-\varepsilon, \varepsilon), l=1, \ldots, 4$, such that

$$
Q^{\sqrt{t}}\left(z^{l}(s), s\right)=0, \quad z^{l}(0)=\mu_{0, \sqrt{t}}^{l} \quad \forall l=1, \ldots, 4 \text { and } \forall s \in(-\varepsilon, \varepsilon) .
$$

Let us look more carefully at the leading term $c_{0, \sqrt{t}}$ of the series expansion $\sum_{j=0}^{\infty} c_{j, \sqrt{t}} t^{j / 2}$ of the remaining four roots. We substitute $\lambda=\lambda(t)=\sum_{j=0}^{\infty} c_{j, \sqrt{t}} t^{j / 2}$ in the characteristic equation (3.3), take the limit $t \rightarrow 0$ and find that $c_{0, \sqrt{t}}$ solves the following equation:

$$
a_{40}\left(c_{0, \sqrt{t}}\right)^{4}+a_{30}\left(c_{0, \sqrt{t}}\right)^{3}+a_{20}\left(c_{0, \sqrt{t}}\right)^{2}+a_{10} c_{0, \sqrt{t}}+a_{00}=0 .
$$

Substituting the coefficients $a_{j 0}, j=0, \ldots, 4$ as in $(3.6 f)-(3.6 b)$ we get that $c_{0, \sqrt{t}}$ solves:

$$
\left[a_{\eta}\left(c_{0, \sqrt{t}}\right)^{2}+2 i k\left(\frac{1}{R_{1}}-\frac{1}{R_{2}}\right) \sin \alpha \cos \alpha c_{0, \sqrt{t}}-k^{2} a_{\xi}\right]^{2}=0 .
$$

Solving this we find the following solutions:

$$
c_{0, \sqrt{t}}=\frac{k}{a_{\eta}}\left[-i\left(\frac{1}{R_{1}}-\frac{1}{R_{2}}\right) \sin \alpha \cos \alpha \pm \frac{1}{\sqrt{R_{1} R_{2}}}\right] .
$$

\subsubsection{Degenerate Cases}

So far, we assumed $a_{\eta} \neq 0$. In many practical situations, however, this is not the case (see, e.g., Figure 2 below with a typical cooling tower shape). If $a_{\eta}=0$ the structure of the layers depends strongly on the geometry of the midsurface, as we shall now show. Accordingly, we distinguish for $a_{\eta}=0$ the hyperbolic and parabolic degeneration (the elliptic case is covered by the basic edge effect). 


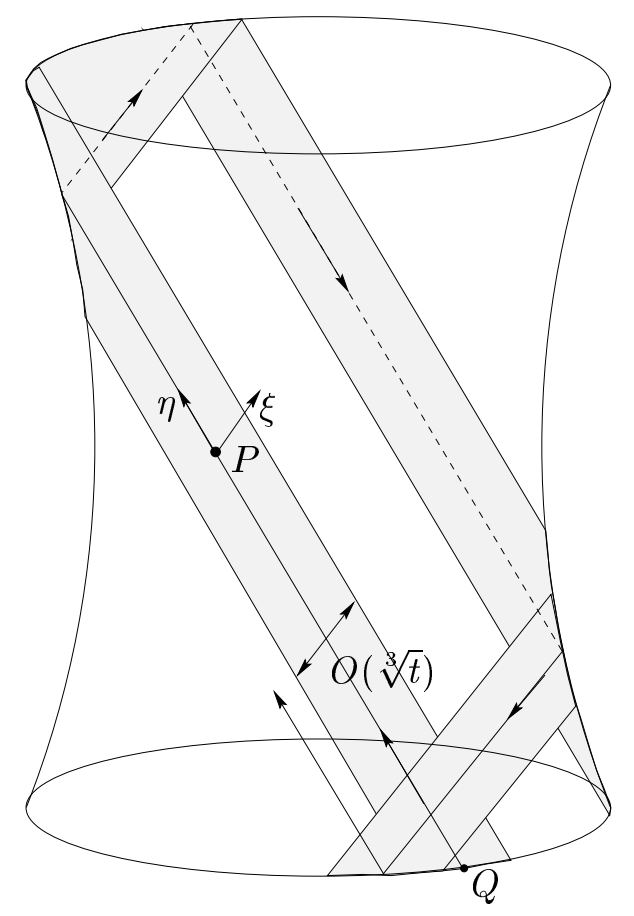

Figure 2: Layer with $L \sim \sqrt[3]{t}$ generated by a propagating singularity at $Q$ on a hyperbolic shell $(O(\sqrt{t})$-layers not shown).

Hyperbolic degeneration. In case of hyperbolic degeneration we have $a_{40}=a_{30}=0$ and $a_{20} \neq 0$ in $(3.6 \mathrm{~b})-(3.6 \mathrm{~d})$. In this case we get a six-fold eigenvalue corresponding to a layer of length scale $L \sim \sqrt[3]{t}$. An interesting consequence of this effect is demonstrated in Figure 2 . It is well known that the membrane theory of hyperbolic shells (i.e., the zero-thickness limit of system (3.1) in our model) is a first order hyperbolic system [31], in which local perturbations propagate in directions where the curvature vanishes, i.e., along the characteristic lines [21]. This is indicated for a source of perturbation at $Q$ in Figure 2. At small, positive thickness, the asymptotic effect is spread out into a layer of width $O(\sqrt[3]{t})$ (the shaded region in Figure 2 ). In our simplified model the characteristic lines are straight, and $\xi=0$ is one of such lines when $a_{\eta}=0$. The precise structure of the layer is then as follows.

Proposition 3.2 Let us assume that the shell is hyperbolic at the point $(x, y)=(0,0)$, i.e., $\tan \alpha= \pm \sqrt{\left|R_{1} / R_{2}\right|}$ and $K:=1 / R_{1} R_{2}<0$. Then six of the roots of the characteristic polynomial take the form $\lambda(t)=z(\sqrt[3]{t}) / \sqrt[3]{t}$, where $z(\cdot)$ is analytic function in a small neighbourhood of 0 . Moreover, the leading term of the series expansion of $z(s)=\sum_{j=0}^{\infty} \mu_{j, \sqrt[3]{t}} s^{j}$ is given by

$$
\mu_{0, \sqrt[3]{t}}=\rho\left(\frac{48\left(1-\nu^{2}\right) k^{2}}{\left|R_{1} R_{2}\right|}\right)^{1 / 6}
$$


with $\rho^{6}-1=0$. This sixfold root of $P_{L N K_{S}}$ corresponds to a short range layer of length scale $O(\sqrt[3]{t})$

The remaining two roots correspond to long range effects and have the form $\lambda(t)=\sum_{j=0}^{\infty} c_{j, \sqrt[3]{t}} t^{j / 3}$, where

$$
c_{0, \sqrt[3]{t}}=-i \frac{k}{2} \sqrt{\left|R_{1} R_{2}\right|}\left(\frac{1}{R_{1}}+\frac{1}{R_{2}}\right) \in i \mathbb{R}
$$

In hyperbolic shells there are therefore always oscillations.

Proof. Let us proceed as in the proof of Proposition 3.1, and make the substitutions $\sqrt[3]{t}=s$, $\lambda=z / s$ in the characteristic equation (3.3). Since $\cos \alpha= \pm \sqrt{\left|R_{2}\right| /\left(\left|R_{1}\right|+\left|R_{2}\right|\right)}$, $\sin \alpha=$ $\pm \sqrt{\left|R_{1}\right| /\left(\left|R_{1}\right|+\left|R_{2}\right|\right)}$, one deduces from $(3.6 b)$, (3.6c) that the coefficients $a_{30}=a_{40}=0$. Multiplying then with $s^{2}$ it follows that $z$ is solution of the equation

$$
\begin{aligned}
Q^{\sqrt[3]{t}}(z, s):= & a_{8}\left(s^{3}\right) z^{8}+s^{7} a_{7}\left(s^{3}\right) z^{7}+s^{2} a_{6}\left(s^{3}\right) z^{6}+s^{3} a_{5}\left(s^{3}\right) z^{5} \\
& +s^{4} \tilde{a}_{4}\left(s^{3}\right) z^{4}+s^{5} \tilde{a}_{3}\left(s^{3}\right) z^{3}+a_{2}\left(s^{3}\right) z^{2}+s a_{1}\left(s^{3}\right) z+s^{2} a_{0}\left(s^{3}\right)=0 .
\end{aligned}
$$

We denoted by $\tilde{a}_{4}(t)=a_{42}+a_{44} t^{2}+a_{46} t^{4}, \tilde{a}_{3}(t)=a_{32}+a_{34} t^{2}+a_{36} t^{4}$. Since we are looking for the leading term $\mu_{0} \sqrt[3]{t}$ of the series expansion with respect to $s$ of the solutions $z=z(s)$, taking the limit $s \rightarrow 0$ in the equation (3.12), it follows that $\mu_{0, \sqrt[3]{t}}$ satisfies

$$
\left(a_{80}\left(\mu_{0, \sqrt[3]{t}}\right)^{6}+a_{20}\right)\left(\mu_{0, \sqrt[3]{t}}\right)^{2}=0 .
$$

For this equation we get 2 trivial and 6 nontrivial solutions, the non-vanishing solutions satisfying

$$
a_{80}\left(\mu_{0, \sqrt[3]{t}}\right)^{6}+a_{20}=0 .
$$

Since for hyperbolic shells the coefficients $a_{20}, a_{80}$ take the following form

$$
a_{20}=2 k^{2} \frac{1}{R_{1} R_{2}}\left(1-\nu^{2}\right)(1-\nu), \quad a_{80}=\frac{1}{24}(1-\nu),
$$

we get easily that

$$
\mu_{0, \sqrt[3]{t}}=\rho\left(\frac{48\left(1-\nu^{2}\right) k^{2}}{\left|R_{1} R_{2}\right|}\right)^{1 / 6}
$$

with $\rho^{6}-1=0$. Let us now denote by $\mu_{0, \sqrt[3]{t}}^{l}, l=1, \ldots, 6$, the solutions of equation (3.13). Then it follows that

$$
Q^{\sqrt[3]{t}}\left(\mu_{0, \sqrt[3]{t}}^{l}, 0\right)=0, \quad \frac{\partial Q^{\sqrt[3]{t}}}{\partial z}\left(\mu_{0, \sqrt[3]{t}}^{l}, 0\right) \neq 0, \quad \text { for } l=1, \ldots, 6 .
$$


Since $Q^{\sqrt[3]{t}}(\cdot, \cdot)$ in (3.12) is analytic function in both variables $(z, s)$, the Implicit Function Theorem implies that there exist $\varepsilon>0$ and $z^{l}(\cdot) \in \mathcal{A}(-\varepsilon, \varepsilon), l=1, \ldots, 6$, such that $Q^{\sqrt[3]{t}}\left(z^{l}(s), s\right)=$ $0, \forall s \in(-\varepsilon, \varepsilon)$ and $z^{l}(0)=\mu_{0, \sqrt[3]{t}}^{l}, l=1, \ldots, 6$.

The leading term $c_{0, \sqrt[3]{t}}$ of the series expansion of the remaining two roots $\lambda=\lambda(t)=$ $\sum_{j=0}^{\infty} c_{j, \sqrt[3]{t}} t^{j / 3}$ solves in this case the equation (recall that here $a_{40}=a_{30}=0$ )

$$
a_{20}\left(c_{0, \sqrt[3]{t}}\right)^{2}+a_{10} c_{0, \sqrt[3]{t}}+a_{00}=0 .
$$

For this particular geometry of the shell $\left(a_{\eta}=0, a_{\xi}=1 / R_{1}+1 / R_{2}\right)$ this equation takes the form:

$$
\left[\left(\frac{1}{R_{1}}+\frac{1}{R_{2}}\right) k-2 i \sqrt{\frac{1}{\left|R_{1} R_{2}\right|}} c_{0, \sqrt[3]{t}}\right]^{2}=0 .
$$

Solving this we obtain (3.11).

We complete the discussion by considering the

Parabolic degeneration. Assume that $a_{\eta}=0$ and that the shell is parabolic at $P$, i.e., either $R_{1}=\infty$ and $\alpha=\pi / 2$ or $R_{2}=\infty$ and $\alpha=0$ (see Figure 3). Then we only get layers with length scale $L \sim \sqrt[4]{t}$, as we see in the following

Proposition 3.3 In the case of parabolic shells the 8 roots of the characteristic polynomial are of the form $\lambda(t)=z(\sqrt[4]{t}) / \sqrt[4]{t}$, with $z(\cdot)$ analytic function in a small neighbourhood of 0 , and the leading term $\mu_{0, \sqrt[4]{t}}$ of the series expansion $z(s)=\sum_{j=0}^{\infty} \mu_{j, \sqrt[4]{t}} s^{j}$ takes the form

$$
\mu_{0, \sqrt[4]{t}}=\rho \sqrt[8]{\frac{12\left(1-\nu^{2}\right) k^{4}}{R^{2}}}
$$

with $\rho^{8}+1=0$ and $R=\min \left\{\left|R_{1}\right|,\left|R_{2}\right|\right\}$.

Remark 3.4 In this way we found all 8 roots, therefore there are no further scales. Note that the length scale $\sqrt[4]{t}$ is, formally, short range, however in practice this edge effect may spread throughout the domain (e.g., for $t=10^{-3}, \sqrt[4]{t} \simeq 0.177$ ).

Proof of Proposition 3.3. Let us first make the substitutions $\lambda=z / s, s=\sqrt[4]{t}$ and transform the characteristic equation (3.3) with respect to the new variable $s$. Since for parabolic shells the coefficients $a_{40}=a_{30}=a_{20}=a_{10}=0$, as one deduces easily from $(3.6 e),(3.6 d),(3.6 c)$, $(3.6 b)$, we find that $z$ solves the following equation

$$
\begin{aligned}
Q^{\sqrt[4]{t}}(z, s):= & a_{8}\left(s^{4}\right) z^{8}+s^{9} a_{7}\left(s^{4}\right) z^{7}+s^{2} a_{6}\left(s^{4}\right) z^{6}+s^{3} a_{5}\left(s^{4}\right) z^{5}+s^{4} \tilde{a}_{4}\left(s^{4}\right) z^{4} \\
& +s^{5} \tilde{a}_{3}\left(s^{4}\right) z^{3}+s^{6} \tilde{a}_{2}\left(s^{4}\right) z^{2}+s^{7} \tilde{a}_{1}\left(s^{4}\right) z+a_{0}\left(s^{4}\right)=0
\end{aligned}
$$




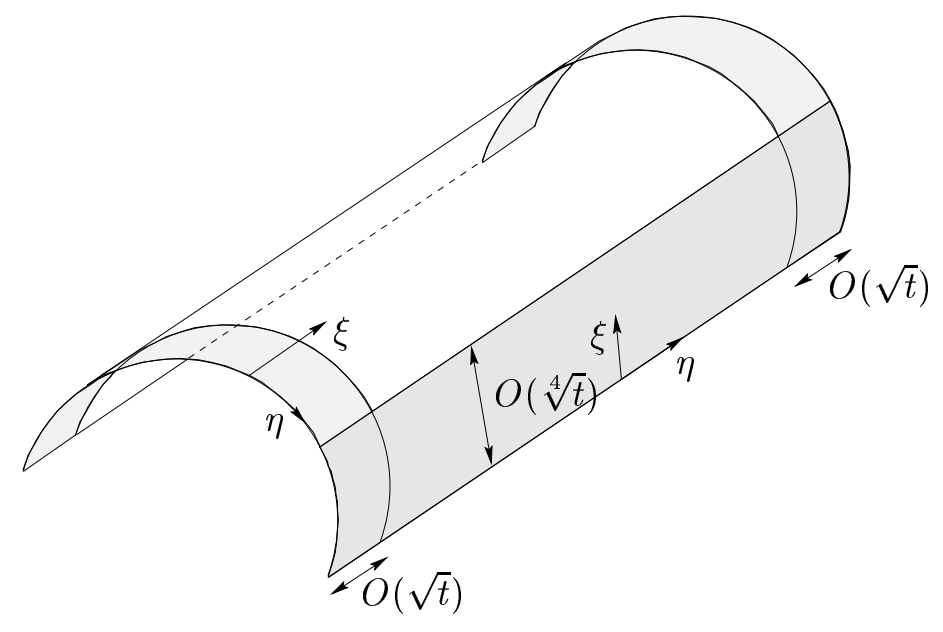

Figure 3: Barrel-Vault with $O(\sqrt{t}), O(\sqrt[4]{t})$ boundary layers

where

$$
\begin{array}{ll}
\tilde{a}_{4}(t)=a_{42}+a_{44} t^{2}+a_{46} t^{4}, & \tilde{a}_{3}(t)=a_{32}+a_{34} t^{2}+a_{36} t^{4}, \\
\tilde{a}_{2}(t)=a_{22}+a_{24} t^{2}+a_{26} t^{4}, & \tilde{a}_{1}(t)=a_{12}+a_{14} t^{2}+a_{16} t^{4} .
\end{array}
$$

Assuming a-priori the series expansion $z(s)=\sum_{j=0}^{\infty} \mu_{j, \sqrt[4]{t}} s^{j}$ for the solution $z$ and taking the limit $s \rightarrow 0$ in (3.15) we find that $\mu_{0, \sqrt[4]{t}}$ satisfies the following equation:

$$
\left(\mu_{0, \sqrt[4]{t}}\right)^{8} a_{80}+a_{00}=0
$$

i.e., $\mu_{0, \sqrt[4]{t}}=\rho \sqrt[8]{a_{00} / a_{80}}$, with $\rho^{8}+1=0$. Since in the case of parabolic shells the coefficients $a_{00}, a_{80}$ take the form

$$
a_{00}=\frac{1}{2} k^{4}(1-\nu) \frac{\left(1-\nu^{2}\right)}{R^{2}}, \quad a_{80}=\frac{1}{24}(1-\nu),
$$

the formula (3.14) for the leading term $\mu_{0, \sqrt[4]{t}}$ follows easily. Let us further denote by $\mu_{0, \sqrt[4]{t}}^{l}$, $l=1, \ldots, 8$, the solutions of the equation (3.16). Since $Q \sqrt[4]{t}(z, s)$ is an analytic function of $(z, s)$ and

$$
Q^{\sqrt[4]{t}}\left(\mu_{0, \sqrt[4]{t}}^{l}, 0\right)=0, \quad \frac{\partial Q^{\sqrt[4]{t}}}{\partial z}\left(\mu_{0, \sqrt[4]{t}}^{l}, 0\right) \neq 0, \quad \text { for } l=1, \ldots, 8
$$

the Implicit Function Theorem implies that there exist $\varepsilon>0$ and $z^{l}(\cdot) \in \mathcal{A}(-\varepsilon, \varepsilon)$, such that $Q^{\sqrt[4]{t}}\left(z^{l}(s), s\right)=0, \forall s \in(-\varepsilon, \varepsilon)$ and $z^{l}(0)=\mu_{0, \sqrt[4]{t}}^{l}$ for $l=1, \ldots, 8$. Consequently, the $\sqrt[4]{t}$-length scale eigenvalues are of the form $\lambda_{l}(t)=z^{l}(\sqrt[4]{t}) / \sqrt[4]{t}, l=1, \ldots, 8$. 


\subsection{The long range Fourier modes}

In Proposition 3.1 we have seen that the characteristic polynomial $P_{\mathrm{LNK}_{\mathrm{S}}}(\lambda)$ has four roots $\lambda=\lambda(t)$ which are bounded as $t \rightarrow 0$. Here we analyze these roots and the corresponding deformation states in more detail. Due to (3.10), we assume throughout this section that the curvature along the $\eta$-axis does not vanish, i.e., $a_{\eta} \neq 0$. The case $a_{\eta}=0$ was discussed in Section 3.1.2.

We analyze the four roots $\lambda=\lambda(t)=\sum_{j=0}^{\infty} c_{j, \sqrt{t}} t^{j / 2}$ of the characteristic polynomial $P_{\mathrm{LNK}_{\mathrm{S}}}(\lambda)$ of the $\mathrm{LNK}_{\mathrm{S}}$ shell model and investigate the dependence of the coefficients $c_{0, \sqrt{t}}$, $c_{1, \sqrt{t}}$ on the mid-surface geometry at $P$ in various, special cases.

As before, inserting $\lambda=\lambda(t)=\sum_{j=0}^{\infty} c_{j, \sqrt{t}} t^{j / 2}$ in the characteristic equation (3.3) and looking at the coefficients of $t^{0}, t^{1 / 2}, t, t^{3 / 2}$ in the resulting expansion we deduce the following equations for $c_{j, \sqrt{t}}(j=0, \ldots, 3)$

$$
\begin{aligned}
& 0=a_{40}\left(c_{0, \sqrt{t}}\right)^{4}+a_{30}\left(c_{0, \sqrt{t}}\right)^{3}+a_{20}\left(c_{0, \sqrt{t}}\right)^{2}+a_{10} c_{0, \sqrt{t}}+a_{00}, \\
& 0=\left[4 a_{40}\left(c_{0, \sqrt{t}}\right)^{3}+3 a_{30}\left(c_{0, \sqrt{t}}\right)^{2}+2 a_{20} c_{0, \sqrt{t}}+a_{10}\right] c_{1, \sqrt{t}} \text {, } \\
& 0=\left[4 a_{40}\left(c_{0, \sqrt{t}}\right)^{3}+3 a_{30}\left(c_{0, \sqrt{t}}\right)^{2}+2 a_{20} c_{0, \sqrt{t}}+a_{10}\right] c_{2, \sqrt{t}} \\
& +\left[6 a_{40}\left(c_{0, \sqrt{t}}\right)^{2}+3 a_{30} c_{0, \sqrt{t}}+a_{20}\right]\left(c_{1, \sqrt{t}}\right)^{2}, \\
& 0=\left[4 a_{40}\left(c_{0, \sqrt{t}}\right)^{3}+3 a_{30}\left(c_{0, \sqrt{t}}\right)^{2}+2 a_{20} c_{0, \sqrt{t}}+a_{10}\right] c_{3, \sqrt{t}} \\
& +2\left[6 a_{40}\left(c_{0, \sqrt{t}}\right)^{2}+3 a_{30} c_{0, \sqrt{t}}+a_{20}\right] c_{1, \sqrt{t}} c_{2, \sqrt{t}} \\
& +\left(4 a_{40} c_{0, \sqrt{t}}+a_{30}\right)\left(c_{1, \sqrt{t}}\right)^{3} \text {. }
\end{aligned}
$$

After looking more carefully at the first equation (3.17), we see that it is a bi-quadratic one and we deduce that the preceding system of equations is equivalent to the following one:

$$
\begin{aligned}
0 & =a_{40}\left(c_{0, \sqrt{t}}\right)^{4}+a_{30}\left(c_{0, \sqrt{t}}\right)^{3}+a_{20}\left(c_{0, \sqrt{t}}\right)^{2}+a_{10} c_{0, \sqrt{t}}+a_{00}, \\
0 & =\left[6 a_{40}\left(c_{0, \sqrt{t}}\right)^{2}+3 a_{30} c_{0, \sqrt{t}}+a_{20}\right]\left(c_{1, \sqrt{t}}\right)^{2}, \\
0 & =\left(4 a_{40} c_{0, \sqrt{t}}+a_{30}\right)\left(c_{1, \sqrt{t}}\right)^{3} .
\end{aligned}
$$

There are essentially four special cases of this system which we discuss separately. 
Case 1a: $a_{\xi} \neq 0$ and $\left|R_{1} R_{2}\right|<\infty$. In this case the system of equations (3.21)-(3.23) has the following solutions:

$$
\begin{aligned}
c_{0, \sqrt{t}} & =\frac{k}{a_{\eta}}\left[-i\left(\frac{1}{R_{1}}-\frac{1}{R_{2}}\right) \sin \alpha \cos \alpha \pm \frac{1}{\sqrt{R_{1} R_{2}}}\right], \\
c_{1, \sqrt{t}} & =0 .
\end{aligned}
$$

An important particular case arises when $R_{1} R_{2}<0$. Then $c_{0, \sqrt{t}} \in i \mathbb{R}$ and we see that we have in this case (to leading order) an oscillating, non-decaying solution of the boundary layer problem (3.1) of the form $\mathbf{u}=\mathbf{u}_{l}^{k}(\xi, \eta)=e^{i k \eta} e^{\left(i \hat{c}_{0}+O(t)\right) \xi} \mathbf{U}$, where $c_{0, \sqrt{t}}=i \hat{c}_{0}$ with

$$
\hat{c}_{0}=\frac{k}{a_{\eta}}\left[-\left(\frac{1}{R_{1}}-\frac{1}{R_{2}}\right) \cos \alpha \sin \alpha \pm \frac{1}{\sqrt{\left|R_{1} R_{2}\right|}}\right] \text {. }
$$

Case 1b: $a_{\xi} \neq 0$ and $\left|R_{1} R_{2}\right|=\infty$. In this case the equation

$$
a_{40}\left(c_{0, \sqrt{t}}\right)^{4}+a_{30}\left(c_{0, \sqrt{t}}\right)^{3}+a_{20}\left(c_{0, \sqrt{t}}\right)^{2}+a_{10} c_{0, \sqrt{t}}+a_{00}=0
$$

has one nonzero root of multiplicity 4 , which satisfies automatically the equations:

$$
\begin{aligned}
& 0=4 a_{40}\left(c_{0, \sqrt{t}}\right)^{3}+3 a_{30}\left(c_{0, \sqrt{t}}\right)^{2}+2 a_{20} c_{0, \sqrt{t}}+a_{10}, \\
& 0=6 a_{40}\left(c_{0, \sqrt{t}}\right)^{2}+3 a_{30} c_{0, \sqrt{t}}+a_{20}, \\
& 0=4 a_{40} c_{0, \sqrt{t}}+a_{30} .
\end{aligned}
$$

Therefore, $c_{0, \sqrt{t}}=i k \tan \alpha$ (if $\left|R_{1}\right|=\infty$ ), or $c_{0, \sqrt{t}}=-i k / \tan \alpha\left(\right.$ if $\left.\left|R_{2}\right|=\infty\right)$. Since $c_{0, \sqrt{t}} \in i \mathbb{R}$ in both situations this implies that the solution of the boundary layer problem (3.1) is an oscillating one, and has the form $\mathbf{u}=\mathbf{u}_{l}^{k}(\xi, \eta)=e^{i k \eta} e^{i k\left(\hat{c}_{0}+O(\sqrt{t})\right) \xi} \mathbf{U}$, where $\hat{c}_{0}=\tan \alpha$ (if $\left|R_{1}\right|=\infty$ ), or $\hat{c}_{0}=-1 / \tan \alpha$ (if $\left|R_{2}\right|=\infty$ ).

In order to find $c_{1, \sqrt{t}}$ we look at the coefficient of $t^{2}$. We get the following equation:

$$
\begin{aligned}
0 & =a_{80}\left(c_{0, \sqrt{t}}\right)^{8}+a_{60}\left(c_{0, \sqrt{t}}\right)^{6}+\left[4 a_{40}\left(c_{0, \sqrt{t}}\right)^{3}+3 a_{30}\left(c_{0, \sqrt{t}}\right)^{2}+2 a_{20} c_{0, \sqrt{t}}+a_{10}\right] c_{4, \sqrt{t}} \\
& +\left[6 a_{40}\left(c_{0, \sqrt{t}}\right)^{2}+3 a_{30} c_{0, \sqrt{t}}+a_{20}\right]\left(2 c_{1, \sqrt{t}} c_{3, \sqrt{t}}+\left(c_{2, \sqrt{t}}\right)^{2}\right) \\
& +3\left(4 a_{40} c_{0, \sqrt{t}}+a_{30}\right)\left(c_{1, \sqrt{t}}\right)^{2} c_{2, \sqrt{t}} \\
& +a_{40}\left(c_{1, \sqrt{t}}\right)^{4}+a_{42}\left(c_{0, \sqrt{t}}\right)^{4}+a_{32}\left(c_{0, \sqrt{t}}\right)^{3}+a_{22}\left(c_{0, \sqrt{t}}\right)^{2}+a_{12} c_{0, \sqrt{t}}+a_{02},
\end{aligned}
$$

from where we get upon comparing coefficients in $\sqrt{t}$ the following equation for $c_{1, \sqrt{t}}$

$$
\begin{aligned}
\left(c_{1, \sqrt{t}}\right)^{4} & =-\left(a_{40}\right)^{-1}\left[a_{80}\left(c_{0, \sqrt{t}}\right)^{8}+a_{60}\left(c_{0, \sqrt{t}}\right)^{6}\right. \\
& \left.+a_{42}\left(c_{0, \sqrt{t}}\right)^{4}+a_{32}\left(c_{0, \sqrt{t}}\right)^{3}+a_{22}\left(c_{0, \sqrt{t}}\right)^{2}+a_{12} c_{0, \sqrt{t}}+a_{02}\right] .
\end{aligned}
$$

Generally, the preceding equation has non-trivial solutions. 
Case $2 a_{\xi}=0$. In this case $a_{\eta}$ takes the form $a_{\eta}=1 / R_{1}+1 / R_{2}, a_{00}=a_{10}=0$ and the characteristic polynomial $P_{\mathrm{LNK}_{\mathrm{S}}}$ becomes

$$
\begin{aligned}
P_{\mathrm{LNK}_{\mathrm{S}}}\left(\lambda ; t, k, \nu, \alpha, R_{1}, R_{2}\right)= & t^{2} a_{8}(t) \lambda^{8}+t^{4} a_{7}(t) \lambda^{7}+t^{2} a_{6}(t) \lambda^{6}+t^{2} a_{5}(t) \lambda^{5} \\
& +a_{4}(t) \lambda^{4}+a_{3}(t) \lambda^{3}+a_{2}(t) \lambda^{2}+t^{2} \tilde{a}_{1}(t) \lambda+t^{2} \tilde{a}_{0}(t),
\end{aligned}
$$

where $\tilde{a}_{10}(t)=a_{12}+a_{14} t^{2}+a_{16} t^{4}, \tilde{a}_{00}(t)=a_{02}+a_{04} t^{2}+a_{06} t^{4}$. The leading term $c_{0, \sqrt{t}}$ solves in this case

$$
a_{40}\left(c_{0, \sqrt{t}}\right)^{4}+a_{30}\left(c_{0, \sqrt{t}}\right)^{3}+a_{20}\left(c_{0, \sqrt{t}}\right)^{2}=0 .
$$

The equation (3.26) has the following solutions:

$$
c_{0, \sqrt{t}}^{(1)}=c_{0, \sqrt{t}}^{(2)}=0, \quad c_{0, \sqrt{t}}^{(3)}=c_{0, \sqrt{t}}^{(4)}=2 i k \frac{1}{\sqrt{\left|R_{1} R_{2}\right|}} \frac{1}{\frac{1}{R_{1}}+\frac{1}{R_{2}}} .
$$

Now there are essentially two distinct cases.

Case 2a: $a_{\xi}=0,\left|R_{1} R_{2}\right|<\infty$. The last two solutions in (3.27) take values in $i \mathbb{R}$, this means that in this case we get again an oscillating, non-decaying solution of the boundary layer problem (3.1). For the first two trivial solutions $c_{0, \sqrt{t}}^{(1)}=c_{0, \sqrt{t}}^{(2)}=0$ (i.e., we get long range layers) let us look further at the coefficient $c_{1, \sqrt{t}}$. We find that $c_{1, \sqrt{t}}$ solves

$$
a_{20}\left(c_{1, \sqrt{t}}\right)^{2}=0
$$

Since in our case

$$
a_{20}=-\frac{1}{2} k^{2} \gamma^{2}\left(1-\nu^{2}\right)(1-\nu)^{3} \frac{1}{R_{1} R_{2}} \neq 0,
$$

it follows that $c_{1, \sqrt{t}}=0$. This means that in this special case we get deformation states of scale $O(1 / t)$ and the solution of (3.1) is of the form $\mathbf{u}=\mathbf{u}_{l}^{k}(\xi, \eta)=e^{i k \eta} e^{\left(c_{2, \sqrt{t}} t+O\left(t^{3 / 2}\right)\right) \xi} \mathbf{U}$. The coefficient $c_{2, \sqrt{t}}$ solves the equation

$$
a_{20}\left(c_{2, \sqrt{t}}\right)^{2}+a_{02}=0
$$

Case 2b: $a_{\xi}=0$ and $\left|R_{1} R_{2}\right|=\infty$. This case, where either $R_{1}=\infty$ and $\alpha=0$ or $R_{2}=\infty$ and $\alpha=\pi / 2$, is common in practice (see Figure 4). Let $R=\min \left\{\left|R_{1}\right|,\left|R_{2}\right|\right\}$. Then $a_{30}=a_{20}=a_{10}=a_{00}=0$ and $c_{1, \sqrt{t}}$ solves

$$
a_{40}\left(c_{1, \sqrt{t}}\right)^{4}+a_{02}=0
$$




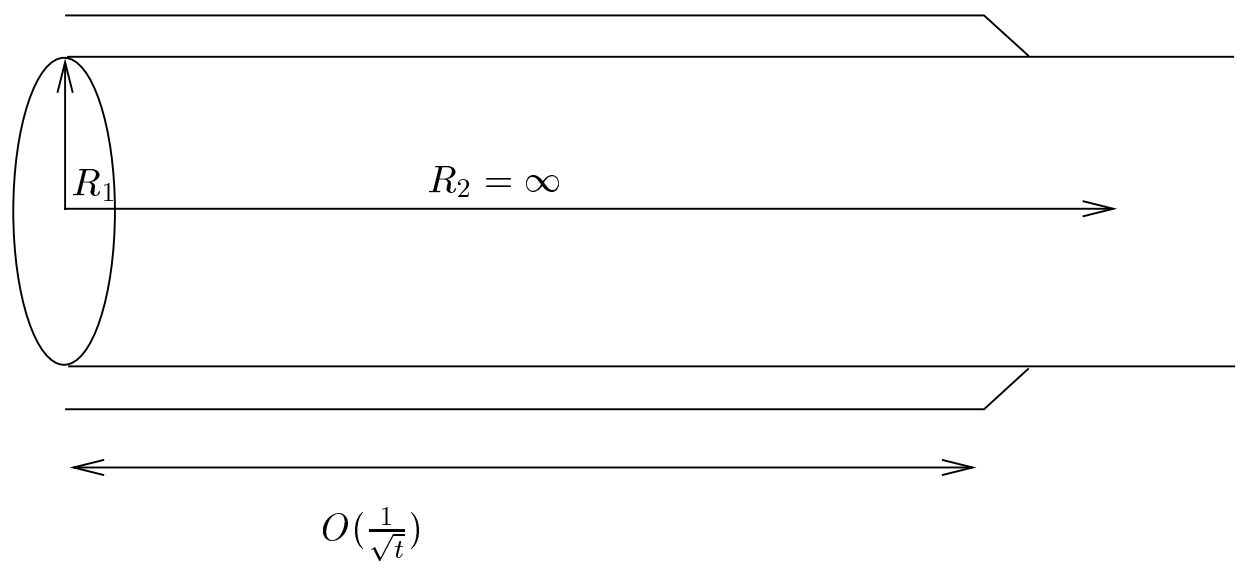

Figure 4: An 'anti-layer' of range $O\left(\frac{1}{\sqrt{t}}\right)$ on a long cylindrical shell.

where the coefficients $a_{02}$ and $a_{40}$ take the form

$$
a_{02}=\frac{1}{24} k^{4}\left[\frac{\left(1-R^{2} k^{2}\right)^{2}}{R^{4}}\right](1-\nu), \quad a_{40}=\frac{1}{2 R^{2}}\left(1-\nu^{2}\right)(1-\nu) .
$$

It follows easily that

$$
c_{1, \sqrt{t}}=\rho \sqrt[4]{\frac{\left(1-R^{2} k^{2}\right)^{2} k^{4}}{12\left(1-\nu^{2}\right) R^{2}}}
$$

where $\rho^{4}+1=0$. Therefore, the solution of the boundary layer problem (3.1) has the form

$$
\mathbf{u}=\mathbf{u}_{l}^{k}(\xi, \eta)=e^{i k \eta} e^{\left(c_{1, \sqrt{t}} \sqrt{t}+O(t)\right) \xi} \mathbf{U}
$$

\subsection{Layer modes in the $\mathrm{RN}_{\mathrm{S}}$-model. The 'plate' layers due to shear- deformation}

So far, we considered shell models without shear. Frequently, in particular with $C^{0}$-finite elements, it is more convenient to consider shell models with shear deformation where the governing equations are second order. This is, in the case of plate bending, the transition from Kirchhoff to Reissner-Mindlin plates. Here, we will show that $a$ ) the shell models with shear deformation exhibit, to leading order, all edge effects of the models without shear, $b$ ) a new short range layer of thickness $O(t)$ arises which, in the plate case, coincides in fact with the layer found by Arnold \& Falk [1].

We start from the homogeneous Euler-Lagrange equations (3.2) for the $\mathrm{RN}_{\mathrm{S}}$ model. Proceeding in the same fashion as for the $\mathrm{LNK}_{\mathrm{S}}$ model with Ansatz (1.2), we get the characteristic equation 


$$
P_{\mathrm{RN}_{\mathrm{S}}}\left(\lambda ; t, k, \nu, \gamma, \alpha, R_{1}, R_{2}\right)=0
$$

where the characteristic polynomial of the Naghdi model for the shallow shell takes the explicit form:

$$
\begin{aligned}
P_{\mathrm{RN}_{\mathrm{S}}}\left(\lambda ; t, k, \nu, \gamma, \alpha, R_{1}, R_{2}\right) & =t^{4} b_{10}(t) \lambda^{10}+t^{6} b_{9}(t) \lambda^{9}+t^{2} b_{8}(t) \lambda^{8}+t^{4} b_{7}(t) \lambda^{7} \\
& +t^{2} b_{6}(t) \lambda^{6}+t^{6} b_{5}(t) \lambda^{5}+b_{4}(t) \lambda^{4}+b_{3}(t) \lambda^{3}+b_{2}(t) \lambda^{2} \\
& +b_{1}(t) \lambda+b_{0}(t)
\end{aligned}
$$

with

$$
\begin{array}{ll}
b_{10}(t)=b_{100}+b_{102} t^{2}, & b_{9}(t)=b_{90}, \\
b_{8}(t)=b_{80}+b_{82} t^{2}+b_{84} t^{4}, & b_{7}(t)=b_{70}+b_{72} t^{2}, \\
b_{6}(t)=b_{60}+b_{62} t^{2}+b_{64} t^{4}, & b_{5}(t)=b_{50}+b_{52} t^{2}+b_{54} t^{4}, \\
b_{4}(t)=b_{40}+b_{42} t^{2}+b_{44} t^{4}+b_{46} t^{6}, & b_{3}(t)=b_{30}+b_{32} t^{2}+b_{34} t^{4}+b_{36} t^{6}, \\
b_{2}(t)=b_{20}+b_{22} t^{2}+b_{24} t^{4}+b_{26} t^{6}, & b_{1}(t)=b_{10}+b_{12} t^{2}+b_{14} t^{4}+b_{16} t^{6}, \\
b_{0}(t)=b_{00}+b_{02} t^{2}+b_{04} t^{4}+b_{06} t^{6} . &
\end{array}
$$

For the boundary layer analysis, the dependence of the following coefficients $b_{i j}$ on the shell geometry will be particularly important:

$$
\begin{aligned}
b_{00} & =-\frac{1}{8} k^{4} \gamma^{2}\left(1-\nu^{2}\right)(1-\nu)^{3}\left(\frac{\cos ^{2} \alpha}{R_{1}}+\frac{\sin ^{2} \alpha}{R_{2}}\right)^{2} \\
b_{10} & =\frac{1}{2} i k^{3} \gamma^{2}\left(1-\nu^{2}\right)(1-\nu)^{3}\left(\frac{1}{R_{1}}-\frac{1}{R_{2}}\right)\left(\frac{\cos ^{2} \alpha}{R_{1}}+\frac{\sin ^{2} \alpha}{R_{2}}\right) \sin \alpha \cos \alpha \\
b_{20} & =\frac{1}{4} k^{2} \gamma^{2}\left(1-\nu^{2}\right)(1-\nu)^{3}\left[3\left(\frac{1}{R_{1}}-\frac{1}{R_{2}}\right)^{2} \sin ^{2} \alpha \cos ^{2} \alpha+\frac{1}{R_{1} R_{2}}\right] \\
b_{30} & =-\frac{1}{2} i k \gamma^{2}\left(1-\nu^{2}\right)(1-\nu)^{3}\left(\frac{1}{R_{1}}-\frac{1}{R_{2}}\right)\left(\frac{\sin ^{2} \alpha}{R_{1}}+\frac{\cos ^{2} \alpha}{R_{2}}\right) \sin \alpha \cos \alpha \\
b_{40} & =-\frac{\gamma^{2}}{8}\left(1-\nu^{2}\right)(1-\nu)^{3}\left(\frac{\sin ^{2} \alpha}{R_{1}}+\frac{\cos ^{2} \alpha}{R_{2}}\right)^{2}, \\
b_{80} & =-\frac{\gamma^{2}}{96}(1-\nu)^{3} \\
b_{100} & =\frac{\gamma}{1152}(1-\nu)^{3} .
\end{aligned}
$$

We consider now the characteristic equation (3.30) and we show first that in (3.30) we cannot get length scales $t^{\beta}$ with $\beta>1$ or $1 / 2<\beta<1$.

Indeed, let us assume that there exist length scales with $\beta>1$. Substituting $\lambda(t)=z\left(t^{\beta}\right) / t^{\beta}$ in the characteristic equation (3.29) and multiplying then the resulting equation with $t^{10 \beta-4}$ 
we get that $z$ satisfies

$$
\begin{aligned}
& b_{10}(t) z^{10}+t^{\beta+2} b_{9}(t) z^{9}+t^{2 \beta-2} b_{8}(t) z^{8}+t^{3 \beta} b_{7}(t) z^{7}+t^{4 \beta-2} b_{6}(t) z^{6}+t^{5 \beta+2} b_{5}(t) z^{5} \\
& +t^{6 \beta-4} b_{4}(t) z^{4}+t^{7 \beta-4} b_{3}(t) z^{3}+t^{8 \beta-4} b_{2}(t) z^{2}+t^{9 \beta-4} b_{1}(t) z+t^{10 \beta-4} b_{0}(t)=0 .
\end{aligned}
$$

Assuming now a-priori that the function $z(\cdot)$ is analytic in a small neighbourhood of 0 and has the expansion $z(s)=\sum_{j=0}^{\infty} \mu_{j, t^{\beta}} s^{j}$, with non-vanishing leading term $\mu_{0, t^{\beta}}$, and taking the limit $t \rightarrow 0$ in (3.32), we find that $\mu_{0, t^{\beta}}$ should satisfy $b_{100}\left(\mu_{0, t^{\beta}}\right)^{10}=0$, which leads to a contradiction, since $b_{100} \neq 0$ implies $\mu_{0, t^{\beta}}=0$.

Analogously, assuming that $1 / 2<\beta<1$, substituting $\lambda=\lambda(t)=z\left(t^{\beta}\right) / t^{\beta}$ in the characteristic equation and multiplying with $t^{8 \beta-2}$ we find the corresponding equation for $z$ :

$$
\begin{aligned}
& t^{2-2 \beta} b_{10}(t) z^{10}+t^{4-\beta} b_{9}(t) z^{9}+b_{8}(t) z^{8}+t^{\beta+2} b_{7}(t) z^{7}+t^{2 \beta} b_{6}(t) z^{6}+t^{3 \beta+4} b_{5}(t) z^{5} \\
& +t^{4 \beta-2} b_{4}(t) z^{4}+t^{5 \beta-2} b_{3}(t) z^{3}+t^{6 \beta-2} b_{2}(t) z^{2}+t^{7 \beta-2} b_{1}(t) z+t^{8 \beta-2} b_{0}(t)=0 .
\end{aligned}
$$

As before, assuming that for such a $\beta$ there exist an analytic function $z(s)=\sum_{j=0}^{\infty} \mu_{j, t^{\beta}} s^{j}$, such that $z\left(t^{\beta}\right)$ solves locally (3.33), and taking the limit $t \rightarrow 0$ in (3.33) we find that the leading term $\mu_{j, t^{\beta}}$ of the expansion should satisfy $b_{80}\left(\mu_{j, t^{\beta}}\right)^{8}=0$. This leads to a contradiction, since $b_{80} \neq 0$. Therefore, there are no length scales $t^{\beta}$ with $\beta \in(1 / 2,1) \cup(1, \infty)$.

Consider now that $\beta=1$. We expect that two of the eigenvalues, those which correspond to the plate layer of length scale $O(t) \sim t$, are of the form $z(t) / t$, with $z(\cdot)$ an analytic function with respect to $t$, for $t \in(-\varepsilon, \varepsilon), \varepsilon>0$ small enough.

Therefore, substituting $\lambda=z / t$ in the characteristic equation (3.29) and multiplying then the equation with $t^{6}$, we find that $z$ solves the equation

$$
\begin{aligned}
Q^{t}(z, t) & :=b_{10}(t) z^{10}+t^{3} b_{9}(t) z^{9}+b_{8}(t) z^{8}+t^{3} b_{7}(t) z^{7}+t^{2} b_{6}(t) z^{6}+t^{7} b_{5}(t) z^{5} \\
& +t^{2} b_{4}(t)+t^{3} b_{3}(t) z^{3}+t^{4} b_{2}(t) z^{2}+t^{5} b_{1}(t) z+t^{6} b_{0}(t)=0
\end{aligned}
$$

We are looking for the leading term $\mu_{0, t}$ of the expansion of the analytic function $z(t)$ in a series with respect to $t$, i.e., $z(t)=\sum_{j=0}^{\infty} \mu_{j, t} t^{j}$. Taking in (3.34) the limit $t \rightarrow 0$, we find that $\mu_{0, t}$ solves

$$
\left(b_{100}\left(\mu_{0, t}\right)^{2}+b_{80}\right)\left(\mu_{0, t}\right)^{8}=0 .
$$

It follows therefore that there are two nontrivial solutions which satisfy $b_{100}\left(\mu_{0, t}\right)^{2}+b_{80}=0$, i.e.,

$$
\mu_{0, t}= \pm \sqrt{-b_{80} / b_{100}}= \pm \sqrt{12 \gamma}
$$

since $b_{80}=-\gamma^{2}(1-\nu)^{3} / 96, b_{100}=\gamma(1-\nu)^{3} / 1152$. This is the length scale found in [1] for plate bending. On the other hand, since $Q^{t}(z, t)$ is an analytic function in both variables and

$$
Q^{t}( \pm \sqrt{12 \gamma}, 0)=0, \frac{\partial Q^{t}}{\partial z}( \pm \sqrt{12 \gamma}, 0) \neq 0
$$


as a simple consequence of the Implicit Function Theorem we find that there exist a small neighbourhood $(-\varepsilon, \varepsilon)$ of 0 and $z_{1}=z_{1}(t), z_{2}=z_{2}(t) \in \mathcal{A}(-\varepsilon, \varepsilon)$, such that $Q^{t}\left(z_{1}(t), t\right)=$ $Q^{t}\left(z_{2}(t), t\right)=0 \forall t \in(-\varepsilon, \varepsilon)$, and $z_{1}(0)=\sqrt{12 \gamma}, z_{2}(0)=-\sqrt{12 \gamma}$.

It remains therefore to consider the remaining 8 roots.

Proposition 3.5 For the $R N_{S}$ shell-models with shear (3.2) we get, to leading order, the same length scales as for $L N K_{S}$ shell model, except the $O(t)$-boundary layers due to shear deformation. In particular, Propositions 3.1, 3.2, 3.3 hold verbatim for the $R N_{S}$ shell.

To see this, we observe that $b_{i 0}=-(1 / 4) \gamma^{2}(1-\nu)^{2} a_{i 0}, i=0,1,2,3,4,8$ to deduce that all the proofs of Propositions 3.1, 3.2, 3.3 carry over to this case.

\section{Full parametric analysis of the layer modes}

In the previous section we investigated the main length scales for the Koiter and Naghdi shallow shell models assuming basically that all parameters, except $t$, are frozen. Having found the basic boundary layer length scales we now move on to what we call the 'full parametric representation'. In this section, we generalize the analysis of Section 3 in the following sense : a) we show that the asymptotic boundary layer behaviour of the shallow shells is faithfully reproduced by the simpler $\mathrm{LNK}_{\mathrm{SS}}$ and $\mathrm{RN}_{\mathrm{SS}}$ models of Section $2, b$ ) we show how the thin shell boundary layers emerge under more general conditions when both $t$ and $k$ are variable parameters.

In the full parametric analysis, we choose for the principal parameters the thickness $t$, the wave number $k$, and the curvature length scale $R$ as defined by (2.6). We introduce also a dimensionless parameter $r$ defined by

$$
r=\left\{\begin{array}{rll}
k t & \text { when } \quad n=1 \\
\sqrt[n]{R k^{2} t} & \text { when } \quad n=2,3,4
\end{array}\right.
$$

where we choose $n=1$ for the 'plate' layer mode $\left(\mathrm{RN}_{\mathrm{S}}\right.$ model $)$ and $n=2,3,4$ for the main layer modes, so that

$$
n= \begin{cases}2 & \text { when } \quad a_{\eta} \neq 0, \\ 3 & \text { in case of hyperbolic degeneration }\left(a_{\eta}=0\right), \\ 4 & \text { in case of parabolic degeneration }\left(a_{\eta}=0\right)\end{cases}
$$

It turns out that the value of $r$ is decisive in the sense that we find the four basic layer modes if and only if $r \ll 1$. We finally introduce parameter $L$ defined for each Fourier mode so that $L \sim 1 /|\operatorname{Re} \lambda|$. When $r \ll 1$, we define this parameter in cases $n=1,2,3,4$ as

$$
L=\frac{r}{k} \quad(r \ll 1, \quad n=1,2,3,4) .
$$

Note that this is consistent with the results of Section 3 above. 


\subsection{Full parametric representation of the characteristic Koiter shell equa- tion}

After an appropriate scaling, the characteristic polynomial (3.3) for the $\mathrm{LNK}_{\mathrm{S}}$ shell becomes

$$
P_{\mathrm{LNK}_{\mathrm{S}}}(\lambda)=P_{\mathrm{LNK}_{\mathrm{SS}}}(\lambda)+P_{1, \mathrm{LNK}_{\mathrm{S}}}(\lambda),
$$

where $P_{\mathrm{LNK}_{\mathrm{SS}}}$ is the characteristic polynomial for the LNK $\mathrm{SS}$ model,

$$
P_{\mathrm{LNK}_{\mathrm{SS}}}(\lambda)=t^{2}\left(\lambda^{2}-k^{2}\right)^{4}+\sum_{m=0, \ldots, 4} b_{m} R^{-2}(i k)^{m} \lambda^{4-m},
$$

and the 'remainder' is of the form

$$
P_{1, \mathrm{LNK}_{\mathrm{S}}}(\lambda)=\sum_{(l, m, p, q) \in \mathcal{I}_{\mathrm{LNK}}} c_{l m p q}^{\mathrm{LNK}_{\mathrm{S}}} t^{p} R^{-q}(i k)^{m} \lambda^{l-m}
$$

The coefficients $b_{m}$ in (4.3) and $c_{l m p q}^{\mathrm{LNK}_{\mathrm{S}}}$ in (4.4) are dimensionless, depend only on the dimensionless parameters $\alpha, \nu$ and

$$
a_{1}=\frac{R}{R_{1}}, \quad a_{2}=\frac{R}{R_{2}}, \quad a_{1}^{2}+a_{2}^{2}=1,
$$

and are uniformly bounded with respect to these parameters. The sum in (4.4) is taken over the indices $(l, m, p, q)$ in the index set $\mathcal{I}_{\mathrm{LNK}_{\mathrm{S}}}$ given by

$$
\mathcal{I}_{\mathrm{LNK}_{\mathrm{S}}}:=\{(l, m, p, q): l \in\{4,6,8\}, m \in\{0, \ldots, l\}, p, q \in\{2,4,6\}, q-p+l=6\},
$$

which ensures that all terms are of same dimension, [length $]^{-6}$ in metric units, since the coefficients $c_{l m p q}^{\mathrm{LNK}_{\mathrm{S}}}$ are dimensionless.

For the shell layers analysis the dependence of the coefficients $\left\{b_{m}\right\}_{m=0, \ldots, 4}$ on the shell geometry at $P$ is decisive. We have

$$
\begin{aligned}
& b_{0}=12\left(1-\nu^{2}\right)\left(a_{1} \sin ^{2} \alpha+a_{2} \cos ^{2} \alpha\right)^{2} \\
& b_{1}=48\left(1-\nu^{2}\right)\left(a_{1} \sin ^{2} \alpha+a_{2} \cos ^{2} \alpha\right) a_{1} a_{2} \sin \alpha \cos \alpha \\
& b_{2}=24\left(1-\nu^{2}\right)\left(3\left(a_{1}-a_{2}\right)^{2} \sin ^{2} \alpha \cos ^{2} \alpha+a_{1} a_{2}\right) \\
& b_{3}=48\left(1-\nu^{2}\right)\left(a_{1} \cos ^{2} \alpha+a_{2} \sin ^{2} \alpha\right)\left(a_{1}-a_{2}\right) \sin \alpha \cos \alpha, \\
& b_{4}=12\left(1-\nu^{2}\right)\left(a_{1} \cos ^{2} \alpha+a_{2} \sin ^{2} \alpha\right)^{2}
\end{aligned}
$$

where $a_{1}, a_{2}$ are defined by (4.5). Note that $b_{m}(m=0, \ldots, 4)$ are, up to a scaling factor, proportional to the coefficients $a_{4-m 0}$ in $(3.6 \mathrm{~b}-3.6 \mathrm{f})$.

We now turn to the analysis of the roots of $P_{\mathrm{LNK}_{\mathrm{S}}}(\lambda)$ in (3.3) in dependence on the parameters $r, R, L$. The analysis reveals three distinct asymptotic behaviours of the roots $\lambda$. Accordingly, we distinguish in the following three parametric cases $(C 1),(C 2)$ and $(C 3)$. 
Case $(C 1) r<<1$ and $L / R<<1$. Here we introduce the (dimensionless) variable $z=\lambda L$ and scale the polynomial $P_{\mathrm{LNK}_{\mathrm{S}}}(\lambda)$ by the factor $K=t^{-2} L^{8}$ to obtain

$$
Q_{\mathrm{LNK}_{\mathrm{S}}}\left(z ; t / L, L / R, r, \nu, \alpha, R_{1} / R\right):=t^{-2} L^{8} P_{\mathrm{LNK}_{\mathrm{S}}}(z / L)
$$

We obtain the full parametric representation of the scaled (Koiter) characteristic equation

$$
Q_{\mathrm{LNK}_{\mathrm{S}}}(z)=Q_{\mathrm{LNK}_{\mathrm{SS}}}(z)+Q_{1, \mathrm{LNK}_{\mathrm{S}}}(z)=0,
$$

where

$$
\begin{aligned}
Q_{\mathrm{LNK}_{\mathrm{SS}}}(z) & =\left(z^{2}-r^{2}\right)^{4}+\sum_{m=0, \ldots, 4} b_{m}\left(\frac{t}{L}\right)^{-2}\left(\frac{L}{R}\right)^{2}(i r)^{m} z^{4-m}, \\
Q_{1, \mathrm{LNK}_{\mathrm{S}}}(z) & =\sum_{(l, m, p, q) \in \mathcal{I}_{\mathrm{LNK}_{\mathrm{S}}}} c_{l m p q}^{\mathrm{LNK}_{\mathrm{S}}}\left(\frac{t}{L}\right)^{p-2}\left(\frac{L}{R}\right)^{q}(i r)^{m} z^{l-m} .
\end{aligned}
$$

Remark 4.1 Note that the scaled leading part $Q_{\mathrm{LNK}_{\mathrm{SS}}}(z)$ is expanded like the remainder $Q_{1, \mathrm{LNK}_{\mathrm{S}}}(z)$, with indices restricted additionally as $p=2, q=0, l=8$ and $m$ even, or $p=$ $0, q=2, l=4$. The scaled expansion is the same as announced before, since

$$
\left(\frac{L}{R}\right)^{2}\left(\frac{t}{L}\right)^{-2} r^{m} z^{l-m}=r^{4-2 n+m} z^{l-m}
$$

We investigate the roots of $Q_{\mathrm{LNK}_{\mathrm{S}}}(z)$ under the condition $(C 1)$. Due to the restrictions (4.6) above, the dominant terms in $Q_{1, \mathrm{LNK}_{\mathrm{S}}}(z)$ come from $m=0$ and are of order $O\left((t / R)^{2}\right)=$ $O\left(r^{2(n-2)}(L / R)^{4}\right)$ (if $\left.l=8\right), O\left((L / R)^{2}\right)$ (if $l=6$ ), or $O\left((L / R)^{4}\right)$ (if $l=4$ ). Therefore, $Q_{1, \mathrm{LNK}_{\mathrm{S}}}(z)$ is, as $r \rightarrow 0$ and $L / R \rightarrow 0$, of order $O\left((L / R)^{2}\right)$. It remains to analyze the dependence of $Q_{\mathrm{LNK}_{\mathrm{SS}}}(z)$ on $r$ and $L / R$. As in Section 3, we distinguish three cases.

The basic case : $a_{1} \sin ^{2} \alpha+a_{2} \cos ^{2} \alpha \neq 0$. Then $b_{0} \neq 0$ and we choose $n=2$. We get that the scaled (Koiter) characteristic polynomial $Q_{\mathrm{LNK}_{\mathrm{S}}}(z)$ takes the form $Q_{\mathrm{LNK}_{\mathrm{S}}}(z)=$ $z^{8}+b_{0} z^{4}+O(i r)+Q_{1, \mathrm{LNK}_{\mathrm{S}}}(z)$. Four of the roots are bounded uniformly in $r$ and $L / R$, as $r \rightarrow 0$ and $L / R \rightarrow 0$, and have the representation

$$
z=\lambda L=z(r, L / R)=\sqrt[4]{b_{0}} \rho+i r z_{1}\left(i r,(L / R)^{2}\right)+(L / R)^{2} z_{2}\left(i r,(L / R)^{2}\right),
$$

where $\rho$ solves $\rho^{4}+1=0$. Here, $z_{j}(\cdot, \cdot)(j=1,2)$ are bounded, analytic functions in both variables and are independent of $i r$ and of $L / R ; b_{0}$ in the absolute term $\sqrt[4]{b_{0}} \rho$ of $z$ is that defined in (4.7a). Note that the $O(\sqrt{t})$ boundary layers are recovered if and only if $r<<1$ and $L / R<<1$, since $\lambda=z / L$ is given by

$$
\lambda=\frac{k z}{r}=\frac{z}{\sqrt{R t}}=\frac{\mu_{0, \sqrt{t}}}{\sqrt{t}}+O(1),
$$

with the leading coefficient $\mu_{0, \sqrt{t}}$ defined in Proposition 3.1. We see from (4.11) that if one or both of the conditions $(C 1)$ is violated, the $O(\sqrt{t})$ layers are lost. 
Hyperbolic degeneration : $a_{1} a_{2}<0$ and $a_{1} \sin ^{2} \alpha+a_{2} \cos ^{2} \alpha=0$. In this case $b_{0}=$ $b_{1}=0$ and $b_{2}=-48\left(1-\nu^{2}\right) a_{1} a_{2}>0$. We choose $n=3$ and the scaled (Koiter) characteristic polynomial becomes

$$
Q_{\mathrm{LNK}_{\mathrm{S}}}(z)=z^{8}+b_{2} z^{2}+O(i r)+Q_{1, \mathrm{LNK}_{\mathrm{S}}}(z)
$$

We obtain therefore a sixfold root which is uniformly bounded with respect to $r$ and $L / R$, given in closed form by

$$
z=z\left(i r,(L / R)^{2}\right)=\sqrt[6]{b_{2}} \rho+i r z_{1}\left(i r,(L / R)^{2}\right)+(L / R)^{2} z_{2}\left(i r,(L / R)^{2}\right),
$$

with $\rho^{6}+1=0$ and generally different functions $z_{j}(\cdot, \cdot), j=1,2$. We see once more that the $O(\sqrt[3]{t})$-layers appear if and only if $r<<1$ and $L / R<<1$ and that then

$$
\lambda=\frac{z}{L}=\frac{k z}{\sqrt[3]{R k^{2} t}}=\frac{\mu_{0, \sqrt[3]{t}}}{\sqrt[3]{t}}+O(1)
$$

with the leading coefficient $\mu_{0}, \sqrt[3]{t}$ as in Proposition 3.2.

Parabolic degeneration : $a_{1} a_{2}=0$ and $a_{1} \sin ^{2} \alpha+a_{2} \cos ^{2} \alpha=0$ (i.e., $a_{1}=0$ and $\alpha=\pi / 2)$. Then $b_{0}=b_{1}=b_{2}=b_{3}=0$ and $b_{4}=12\left(1-\nu^{2}\right) b^{2}>0$. We choose $n=4$ and the (scaled) characteristic polynomial becomes $Q_{\mathrm{LNK}_{\mathrm{S}}}(z)=z^{8}+b_{4}+Q_{1, \mathrm{LNK}_{\mathrm{S}}}(z)$. All the roots are, as $r \rightarrow 0$ and $L / R \rightarrow 0$, uniformly bounded, and can be represented as

$$
z=\sqrt[8]{b_{4}} \rho+(L / R)^{2} z_{1}\left(i r,(L / R)^{2}\right)
$$

with $\rho^{8}+1=0 ; z_{1}(\cdot, \cdot)$ is again a bounded, analytic function which is independent of $r, L / R$. In the parabolic case, we see again that the $O(\sqrt[4]{t})$-layers are present if and only if $(C 1)$ holds, since here $\lambda=z / L$ takes the form

$$
\lambda=\frac{k z}{\sqrt[4]{R k^{2} t}}=\frac{\mu_{0, \sqrt[4]{t}}}{\sqrt[4]{t}}+O(1)
$$

We have seen that $a$ ) the boundary layer length scales found in Section 3 from $P_{\mathrm{LNK}}(\lambda)$ for $t \rightarrow 0$ emerge under more general conditions $(C 1)$, and $b)$ the boundary layer behaviour is, in all cases, to leading order contained in the mathematical Koiter shell.

We will now embark on an analysis of the length scales $\lambda$ in the case when $(C 1)$ is violated. This gives asymptotics that are different from the one in Section 3. We consider first

Case $(C 2) r \sim 1$ or $r>>1(n=2,3,4)$ and $k t<<1$. In this case we set $L=k^{-1}$, $z=\lambda / k$ and scale the characteristic polynomial $P_{\mathrm{LNK}_{\mathrm{S}}}(\lambda)$ by the factor $K=t^{-2} k^{-8}$. We look for bounded (in terms of $1 / r$ and $t / L$ ) roots of the scaled (Koiter) characteristic polynomial

$$
Q_{\mathrm{LNK}_{\mathrm{S}}}(z):=t^{-2} k^{-8} P_{\mathrm{LNK}_{\mathrm{S}}}(k z)=Q_{\mathrm{LNK}_{\mathrm{SS}}}(z)+Q_{1, \mathrm{LNK}_{\mathrm{S}}}(z),
$$


where now

$$
\begin{aligned}
Q_{\mathrm{LNK}_{\mathrm{SS}}}(z) & =\left(z^{2}-1\right)^{4}+\sum_{m=0, \ldots, 4} b_{m} i^{m} r^{-2 n} z^{4-m} \\
Q_{1, \mathrm{LNK}_{\mathrm{S}}}(z) & =\sum_{(l, m, p, q) \in \mathcal{I}_{\mathrm{LNK}_{\mathrm{S}}}} c_{l m p q}^{\mathrm{LNK}_{\mathrm{S}}} i^{m}(t k)^{p-2}(k R)^{q} z^{l-m} \\
& =\sum_{(l, m, p, q) \in \mathcal{I}_{\mathrm{LNK}_{\mathrm{S}}}} c_{l m p q}^{\mathrm{LNK}} i^{m} r^{p-2-q(n-1)}(t / L)^{p-2+q} z^{l-m} .
\end{aligned}
$$

The sum in $Q_{\mathrm{LNK}_{\mathrm{SS}}}(z)$ is of order $O\left(r^{-4}\right)$, whereas in $Q_{1, \mathrm{LNK}_{\mathrm{S}}}(z)$, due to the restrictions (4.6), the dominant terms come from $l=8, q=2, p=4$ and $l=6, p=q=2$. These terms are of order $O\left(r^{4-2 n}(t / L)^{4}\right), O\left(r^{-2}(t / L)^{2}\right)$, respectively. Therefore, in this case

$$
Q_{\mathrm{LNK}_{\mathrm{S}}}(z)=\left(z^{2}-1\right)^{4}+O\left((t / L)^{4}\right)+O\left(r^{-2}(t / L)^{2}\right)+O\left(r^{-4}\right),
$$

and all the roots are bounded as $r \rightarrow \infty$ and $t / L \rightarrow 0$. This means that all the roots $\lambda=k z$ of the (Koiter) characteristic polynomial $P_{\mathrm{LNK}_{\mathrm{S}}}(\lambda)$ scale as $\sim k$ as $r \rightarrow \infty$. Moreover,

$$
Q_{\mathrm{LNK}_{\mathrm{S}}}(z) \rightarrow\left(z^{2}-1\right)^{4}, \quad \text { as } r \rightarrow \infty,
$$

the curvature effects disappear and the limit Fourier mode behaves like a decoupled membrane and plate.

Case $(C 3) r \sim k R$ or $r \gg k R \quad(n=2,3,4)$ and $t / R \ll 1 . \quad$ Since $r=(k R)^{2 / n}(t / R)^{1 / n} \ll$ $(k R)^{2 / n}$, this assumption can hold only in the two degenerate cases $(n=3,4)$ when $k R$ is very small. Assuming this, we define $L=R, z=R \lambda$ and scale the characteristic polynomial by the factor $K=t^{-2} R^{8}$. We then find that the coefficients of the scaled polynomial are uniformly bounded, hence the roots as well. The 'layer' in this case corresponds simply to a smooth deformation.

Of particular interest is the geometry of an infinitely long cylindrical shell (radius $=R$ ) where $\eta$ is chosen as the axial coordinate, so that $n=4$ in (4.1). In this case, when $k$ varies in the range $0 \leq k \ll t^{-1}$, we can sum up our analysis from $(C 1)-(C 3)$ as

$$
L \sim\left\{\begin{array}{rll}
k^{-1} & \text { when }(C 2) & k R \gg(R / t)^{1 / 2} \text { or } k R \sim(R / t)^{1 / 2}, \\
\left(R t / k^{2}\right)^{1 / 4} & \text { when }(C 1) & (R / t)^{1 / 2} \gg k R \gg(t / R)^{1 / 2} \\
R & \text { when }(C 3) & k R \sim(t / R)^{1 / 2} \quad \text { or } k R \ll(t / R)^{1 / 2} .
\end{array}\right.
$$

Note that we again find a true layer mode only in case $(C 1)$, otherwise the 'layer' corresponds to a smooth deformation (case $(C 3)$ ) or a Fourier component of such (case $(C 2)$ ). At the limit $k R \rightarrow 0$ in case $(C 3)$, the deformation obviously approaches that of an arch. 


\subsection{Full parametric representation for the Naghdi shell}

Let us first rewrite the leading term in the Koiter polynomial $P_{\mathrm{LNK}}(\lambda)$, corresponding to the characteristic polynomial for the $\mathrm{LNK}_{\mathrm{SS}}$ model, in the following form

$$
P_{\mathrm{LNK}_{\mathrm{SS}}}(\lambda)=t^{2}\left(\lambda^{2}-k^{2}\right)^{4}+(1-\nu) P_{0, \mathrm{LNK}_{\mathrm{SS}}}(\lambda) .
$$

Then the characteristic polynomial $P_{\mathrm{RN}_{\mathrm{S}}}(\lambda)$ of the $\mathrm{RN}_{\mathrm{S}}$ shallow shell model with shear, when properly scaled, takes the form

$$
\begin{aligned}
P_{\mathrm{RN}_{\mathrm{S}}}(\lambda)= & -\frac{1}{12 \gamma} t^{4}\left(\lambda^{2}-k^{2}\right)^{5}+P_{\mathrm{LNK}_{\mathrm{SS}}}(\lambda) \\
& +\left[-\frac{3-\nu}{12 \gamma} t^{2}\left(\lambda^{2}-k^{2}\right)+\frac{1}{72 \gamma^{2}} t^{4}\left(\lambda^{2}-k^{2}\right)^{2}\right] P_{0, \mathrm{LNK}_{\mathrm{SS}}}(\lambda)+P_{1, \mathrm{RN}_{\mathrm{S}}}(\lambda) .
\end{aligned}
$$

Here the first three terms constitute the characteristic polynomial of the $\mathrm{RN}_{\mathrm{SS}}$ model, and the remainder $P_{1, \mathrm{RN}_{\mathrm{S}}}(\lambda)$ can be again expressed as

$$
P_{1, \mathrm{RN}_{\mathrm{S}}}(\lambda)=\sum_{(l, m, p, q) \in \mathcal{I}_{\mathrm{RN}_{\mathrm{S}}}} c_{l m p q}^{\mathrm{RN}_{\mathrm{S}}} t^{p} R^{-q}(i k)^{m} \lambda^{l-m},
$$

where now the sum is taken over the indices $(l, m, p, q)$ in the index set $\mathcal{I}_{\mathrm{RN}_{\mathrm{S}}}$

$\mathcal{I}_{\mathrm{RN}_{\mathrm{S}}}:=\{(l, m, p, q): l \in\{4,6,8,10\}, m \in\{0, \ldots, l\}, p \in\{2,4,6,8\}, q \in\{2,4,6\}, q-p+l=6\}$ and the coefficients $c_{l m p q}^{\mathrm{RN}_{\mathrm{S}}}$ are quadratic polynomials of $1 / \gamma$.

Case $(C 1) r<<1$ and $L / R<<1$. Here we take again $z=\lambda L$ and scale the Naghdi characteristic polynomial $P_{\mathrm{RN}_{\mathrm{S}}}(\lambda)$ in (4.15) by the factor $t^{-2} L^{8}$

$$
\begin{aligned}
& Q_{\mathrm{RN}_{\mathrm{S}}}(z):=t^{-2} L^{8} P_{\mathrm{RN}_{\mathrm{S}}}(z / L) \\
= & -\frac{1}{12 \gamma}\left(\frac{t}{L}\right)^{2}\left(z^{2}-r^{2}\right)^{5}+\left(z^{2}-r^{2}\right)^{4}+\sum_{m=0, \ldots, 4} b_{m}\left(\frac{t}{L}\right)^{-2}\left(\frac{L}{R}\right)^{2}(i r)^{m} z^{4-m} \\
+ & {\left[-\frac{3-\nu}{12 \gamma}\left(\frac{t}{L}\right)^{2}\left(z^{2}-r^{2}\right)+\frac{1}{72 \gamma^{2}}\left(\frac{t}{L}\right)^{4}\left(z^{2}-r^{2}\right)^{2}\right]\left(\sum_{m=0, \ldots, 4} \tilde{b}_{m}\left(\frac{t}{L}\right)^{-2}\left(\frac{L}{R}\right)^{2}(i r)^{m} z^{4-m}\right) } \\
+ & \sum_{(l, m, p, q) \in \mathcal{I}_{\mathrm{RN}_{\mathrm{S}}}} c_{l m p q}^{\mathrm{RN}_{\mathrm{S}}}\left(\frac{t}{L}\right)^{p-2}\left(\frac{L}{R}\right)^{q}(i r)^{m} z^{l-m},
\end{aligned}
$$

where $\tilde{b}_{m}=b_{m} /(1-\nu)$. Now, since $t / L=r^{n-2} L / R$, if we take $n=2,3,4$ it follows that $t / L<<1$ and we clearly see from (4.16) that the gap between the scaled Koiter characteristic polynomial $Q_{\mathrm{LNK}_{\mathrm{S}}}(z)$ in (4.8) and the scaled Naghdi characteristic polynomial $Q_{\mathrm{RN}_{\mathrm{S}}}(z)$ in (4.16) is of order $O\left((t / L)^{2}\right)+O\left((L / R)^{2}\right)$, which allows to apply the analysis from (4.11)(4.13). 
From (4.16) we find also the short-range 'plate' layer modes, since (4.16) remains valid when choosing $r, L$ according to (4.1)-(4.2) with $n=1$. We find then a twofold root $z$ which is uniformly bounded with respect to $r$ and $L / R$. Moreover, $z=z(i r, L / R)$ is analytic in $r$ and $L / R$ and has for small $r$ and $L / R$ the asymptotic form

$$
z=z(i r, L / R)= \pm \sqrt{12 \gamma} \frac{L}{t}+O\left(r^{2}\right)+O\left((L / R)^{2}\right) .
$$

Note that according to (4.16) the $O(t)$ 'plate' layers in Section 3 are recovered if and only if $r<<1$ and $L / R<<1$ and that $\lambda=z / L$ is given by

$$
\lambda= \pm \frac{\sqrt{12 \gamma}}{t}+O(1)=\frac{\mu_{0, t}}{t}+O(1)
$$

with the leading term $\mu_{0, t}= \pm \sqrt{12 \gamma}$ from (3.35).

Case $(C 2) r \sim 1$ or $r>>1 \quad(n=2,3,4)$. We introduce again the variable $z:=\lambda / k$ and scale the Naghdi characteristic polynomial $P_{\mathrm{RN}_{\mathrm{S}}}(\lambda)$ by the factor $t^{-2} k^{-8}$, resulting in

$$
\begin{aligned}
& Q_{\mathrm{RN}_{\mathrm{S}}}(z):=t^{-2} k^{-8} P_{\mathrm{RN}_{\mathrm{S}}}(k z) \\
& =-\frac{1}{12 \gamma}(k t)^{2}\left(z^{2}-1\right)^{5}+\left(z^{2}-1\right)^{4}+\sum_{m=0, \ldots, 4} b_{m} i^{m} r^{-2 n} z^{4-m} \\
& +\left[-\frac{3-\nu}{12 \gamma}(k t)^{2}\left(z^{2}-1\right)+\frac{1}{72 \gamma^{2}}(k t)^{4}\left(z^{2}-1\right)^{2}\right]\left(\sum_{m=0, \ldots, 4} \tilde{b}_{m} i^{m} r^{-2 n} z^{4-m}\right) \\
& +\sum_{(l, m, p, q) \in \mathcal{I}_{\mathrm{RN}_{\mathrm{S}}}} c_{l m p q}^{\mathrm{RN}_{\mathrm{S}}} i^{m} r^{-q n}(k t)^{p-2+q} z^{l-m} .
\end{aligned}
$$

Assuming $k t \ll 1$ we see that the gap between the scaled Koiter characteristic polynomial $Q_{\mathrm{LNK}_{\mathrm{S}}}(z)$ in (4.14) and the scaled Naghdi polynomial $Q_{\mathrm{RN}_{\mathrm{S}}}(z)$ in $(4.17)$ is of order $O\left((k t)^{2}\right)$, which allows to apply the analysis from the previous section.

\section{$5 \quad$ Energy view on the layer modes}

In this final section we take a step towards modernism. The problem is to find a scaled energy expression that a finite element solver must face when trying to resolve a given layer mode by minimizing energy. Although we know that the energy to be minimized must be the usual strain energy (2.1) or (2.18), there remains a scaling problem since the layer mode appears in parametric length scales and since the relative amplitudes of the different displacement components in the mode are parametric as well. The idea is to scale off this parametric dependence so that the layer mode can be viewed as a smooth, (essentially) non-parametric function that varies in the unit range, regarding both the variables and the amplitudes. After such a scaling, the strain energy density in turn takes a parametric form, and this is our 
target. From the parametric energy density one can further analyze error amplification or locking effects that may occur in finite element modelling.

Below we derive the scaled energy densities for the four basic layer modes found in the previous sections. Thus we assume that in the $\eta$-direction the length scale of the layer mode is set by the Fourier wave number $k$ and that in the $\xi$-direction the length scale is one of the four options where $L \sim t^{1 / n}, n=1,2,3,4$. We keep both $t$ and $k$ as active parameters in this study.

Below we will treat only the simpler variant $\mathrm{RN}_{\mathrm{SS}}$ of the two shallow $\mathrm{RN}$ models, as it turns out that this model is sufficient for extracting the main parametric effects. The results could be extended easily to the $\mathrm{RN}_{\mathrm{S}}$ model. We perform the analysis in the rotated coordinates $\xi, \eta$. For simplicity, we rewrite these coordinates now as $x, y$ and redefine $u, v$ and $\theta, \psi$ from this on as the rotated components of the tangential displacement \& rotation. The transverse shear and bending strains in the $\mathrm{RN}_{\mathrm{SS}}$-model are then still defined by (2.21), but the membrane strains (2.14) need to be rotated so that we get

$$
\beta_{11}=\frac{\partial u}{\partial x}+\frac{a}{R} w, \quad \beta_{22}=\frac{\partial v}{\partial y}+\frac{b}{R} w, \quad \beta_{12}=\frac{1}{2}\left(\frac{\partial u}{\partial y}+\frac{\partial v}{\partial x}\right)+\frac{c}{R} w .
$$

Here we introduce the rotated dimensionless curvatures $a=R a_{\xi}, b=R a_{\eta}$ and $c=R a_{\xi \eta}$, where $R$ is the curvature length scale as before, $a_{\xi}$ and $a_{\eta}$ are defined by (3.7), and

$$
a_{\xi \eta}=\left(\frac{1}{R_{2}}-\frac{1}{R_{1}}\right) \cos \alpha \sin \alpha
$$

\section{$5.1 \quad$ Scaled energy densities}

The main task is to find the relative amplitudes of the displacements and rotations in the layer mode. We proceed from the Euler equations (3.2) (the last terms in the first two equations dropped), observing that $\partial / \partial x$ and $\partial / \partial y$ correspond to amplitude scaling by $L^{-1}$ and $k$, respectively.

Our primary interest is in the main layer modes where $L$ is given by (4.1) - (4.2) with $n \in\{2,3,4\}$ and $r \ll 1$. Starting from the last two equations in (3.2) we first conclude that the amplitudes of $\rho_{1}, \rho_{2}$ must be related to those of $R \kappa_{i j}$ as

$$
\begin{aligned}
& R L t^{-2} \rho_{1} \sim R\left[\kappa_{11}+\nu \kappa_{22}+(1-\nu) r \kappa_{12}\right], \\
& R L t^{-2} \rho_{2} \sim R\left[r\left(\kappa_{11}+\nu \kappa_{22}\right)+(1-\nu) \kappa_{12}\right] .
\end{aligned}
$$

Here $R L t^{-2} \gg 1$, so the amplitudes of $\rho_{1}$ and $\rho_{2}$ must be relatively small. Then we must have $\theta \approx \partial w / \partial x$ and $\psi \approx \partial w / \partial y$, hence $\theta \sim L^{-1} w$ and $\psi \sim k w$, and thus further

$$
\kappa_{11} \sim L^{-2} w, \quad \kappa_{22} \sim L^{-2} r^{2} w, \quad \kappa_{12} \sim L^{-2} r w
$$

and

$$
\rho_{1} \sim t^{2} L^{-3} w, \quad \rho_{2} \sim t^{2} k L^{-2} w
$$


We may now scale the energy density due to transverse shear and bending deformations: Upon rescaling the coordinates and the rotations as

$$
x \hookrightarrow L x, \quad y \hookrightarrow k^{-1} y, \quad \theta \hookrightarrow L^{-1} \theta, \quad \psi \hookrightarrow k \psi,
$$

the new coordinates are dimensionless, varying in the unit range, and the amplitude range of the scaled rotations is normalized to be that of $w$. We scale also the energy density by factor $24 t^{-2} L^{4}$ so that the coefficient of the dominant term $\kappa_{11}^{2}$ is normalized to unity. The resulting scaled expression of the combined transverse shear and bending energy density is then written as

$$
\begin{aligned}
f_{s b}(\mathbf{u}) & =6(1-\nu) \gamma\left(\frac{L}{t}\right)^{2}\left[\left(\theta-\frac{\partial w}{\partial x}\right)^{2}+r^{2}\left(\psi-\frac{\partial w}{\partial y}\right)^{2}\right] \\
& +\nu\left(\frac{\partial \theta}{\partial x}+r^{2} \frac{\partial \psi}{\partial y}\right)^{2}+(1-\nu)\left(\frac{\partial \theta}{\partial x}\right)^{2}+\frac{1}{2}(1-\nu) r^{2}\left(\frac{\partial \theta}{\partial y}+\frac{\partial \psi}{\partial x}\right)^{2} \\
& +(1-\nu) r^{4}\left(\frac{\partial \psi}{\partial y}\right)^{2} \quad(n=2,3,4) .
\end{aligned}
$$

To find the scaled expression of the membrane energy density for the main layer modes, we study the first three equations in (3.2). By the results so far, we know that $\partial \rho_{1} / \partial x+\partial \rho_{2} / \partial y \sim$ $t^{2} L^{-4} w$ there. We will rearrange the membrane energy density in this study as

$$
f_{m}(\mathbf{u})=12 t^{-2} L^{4}\left[\left(\beta_{11}+\nu \beta_{22}\right)^{2}+\left(1-\nu^{2}\right) \beta_{22}^{2}+2(1-\nu) \beta_{12}^{2}\right],
$$

where we also inserted the above scaling factor in front. We consider the three cases $n=2,3,4$ separately.

First assume that $b \neq 0$, so that $n=2$. In this case, viewing the first three equations in (3.2) as a system for the unknowns $\beta_{11}+\nu \beta_{22}, \nu \beta_{11}+\beta_{22}, \beta_{12}$, we find easily that the amplitude scaling must be

$$
\beta_{11}+\nu \beta_{22} \sim r^{2} R^{-1} w, \quad \nu \beta_{11}+\beta_{22} \sim R^{-1} w, \quad \beta_{12} \sim r R^{-1} w .
$$

Together with (5.1) this further implies that with the rescaling

$$
u \hookrightarrow\left\{\begin{array} { r l } 
{ L R ^ { - 1 } u , } & { \text { if } a + \nu b \neq 0 , } \\
{ r L R ^ { - 1 } u , } & { \text { if } a + \nu b = 0 , }
\end{array} \quad v \hookrightarrow \left\{\begin{array}{ccc}
L R^{-1} v, & \text { if } c \neq 0 \\
r L R^{-1} v, & \text { if } \quad c=0
\end{array}\right.\right.
$$

the amplitudes of $u, v, w$ vary in the same range, except for the special case where either $a=\nu=0$ or $a+\nu b=0$ and $c=0$, in which case the rescaling of $u$ should be $u \hookrightarrow r^{2} L R^{-1} u$. Noting finally that $t^{-2} L^{4}=R^{2}$ when $n=2$, we can rewrite (5.3) in case $a+\nu b \neq 0$ explicitly as

$$
\begin{aligned}
f_{m}(\mathbf{u}) & =12\left(\frac{\partial u}{\partial x}+(a+\nu b) w+r \nu \frac{\partial v}{\partial y}\right)^{2}+12\left(1-\nu^{2}\right)\left(r \frac{\partial v}{\partial y}+b w\right)^{2} \\
& +6(1-\nu)\left(r \frac{\partial u}{\partial y}+\frac{\partial v}{\partial x}+2 c w\right)^{2} \quad(n=2, a+\nu b \neq 0, c \neq 0)
\end{aligned}
$$


or as

$$
\begin{aligned}
f_{m}(\mathbf{u}) & =12\left(\frac{\partial u}{\partial x}+(a+\nu b) w+r^{2} \nu \frac{\partial v}{\partial y}\right)^{2}+12\left(1-\nu^{2}\right)\left(r^{2} \frac{\partial v}{\partial y}+b w\right)^{2} \\
& +6(1-\nu) r^{2}\left(\frac{\partial u}{\partial y}+\frac{\partial v}{\partial x}\right)^{2} \quad(n=2, a+\nu b \neq 0, c=0) .
\end{aligned}
$$

When $a+\nu b=0,(5.4)-(5.5)$ hold with $r u$ replacing $u$, except for the mentioned special cases where $r^{2} u$ should replace $u$.

Next consider the case of hyperbolic degeneration where $b=0, c= \pm R\left(-R_{1} R_{2}\right)^{-1 / 2} \neq 0$, and $n=3$. In this case we find from (3.2) that

$$
\beta_{11}+\nu \beta_{22} \sim r^{3} R^{-1} w, \quad \nu \beta_{11}+\beta_{22} \sim r R^{-1} w, \quad \beta_{12} \sim r^{2} R^{-1} w
$$

and from these further that

$$
u-\frac{a}{2 c} v \doteqdot \vartheta \sim r L R^{-1} w, \quad v \sim L R^{-1} w .
$$

Note that here $v$ and $\vartheta$ are displacement components in the two characteristic directions where the curvature vanishes. Using now these as the components of the tangential displacement vector, with the amplitudes rescaled as

$$
\vartheta \hookrightarrow r L R^{-1} \vartheta, \quad v \hookrightarrow L R^{-1} v
$$

and finally noting that $t^{-2} L^{4}=R^{2} r^{-2}$ when $n=3$, we find that in case of hyperbolic degeneration

$$
\begin{aligned}
f_{m}(\mathbf{u}) & =12 r^{-2}\left[r \frac{\partial \vartheta}{\partial x}+\frac{a}{2 c}\left(\frac{\partial v}{\partial x}+2 c w\right)+r \nu \frac{\partial v}{\partial y}\right]^{2}+12\left(1-\nu^{2}\right)\left(\frac{\partial v}{\partial y}\right)^{2} \\
& +6 r^{-2}(1-\nu)\left(r^{2} \frac{\partial \vartheta}{\partial y}+r \frac{a}{2 c} \frac{\partial v}{\partial y}+\frac{\partial v}{\partial x}+2 c w\right)^{2} \quad(n=3) .
\end{aligned}
$$

Finally in case of parabolic degeneration where $b=c=0, a \neq 0, n=4$, we find in a similar way the scaling

$$
\beta_{11}+\nu \beta_{22} \sim r^{4} R^{-1} w, \quad \nu \beta_{11}+\beta_{22} \sim r^{2} R^{-1} w, \quad \beta_{12} \sim r^{3} R^{-1} w
$$

the consistent rescaling

$$
u \hookrightarrow k^{-1} r R^{-1} u, \quad v \hookrightarrow k^{-1} r^{2} R^{-1} v,
$$

and, after the final scaling with $t^{-2} L^{4}=R^{2} r^{-4}$, the scaled energy density

$$
\begin{aligned}
f_{m}(\mathbf{u}) & =12 r^{-4}\left(\frac{\partial u}{\partial x}+a w+r^{2} \nu \frac{\partial v}{\partial y}\right)^{2}+12\left(1-\nu^{2}\right)\left(\frac{\partial v}{\partial y}\right)^{2} \\
& +6 r^{-2}(1-\nu)\left(\frac{\partial u}{\partial y}+\frac{\partial v}{\partial x}\right)^{2} \quad(n=4) .
\end{aligned}
$$


Remark 5.1 The above analysis is easily extended to the case where $r \sim 1$ or $r \gg 1$ and $k t \ll 1$ (Case $(\mathrm{C} 2)$ in Section 4.1). In this parametric range we find that expression (5.2) holds with $L=k^{-1}$ and $r=1$ as expected. Thus the shear and bending energy densities are both isotropic in this range and correspond actually to the Reissner-Mindlin plate-bending model for a plate of effective thickness $t_{\text {eff }} \sim R t / L \sim R k t$. As far as the membrane energy is concerned, the coordinate rescaling $x \hookrightarrow k x$ and the corresponding consistent amplitude rescaling $u \hookrightarrow(k R)^{-1} u, v \hookrightarrow(k R)^{-1} v$ produce the additional factor $\left(R k^{2} t\right)^{-2}=r^{-2 n}$ in front, otherwise the expressions (5.4)-(5.7) are valid with $r=1$. Thus there is no dependence on $n$ in this range, and apart from the small scaling factor in front, the membrane energy density takes the usual non-parametric form.

It remains to consider the case $n=1$. This short-range layer mode (studied already in [1]) is more specific, since it turns out that for this mode $u=v=w=0$ and also $\partial \theta / \partial x+\partial \psi / \partial y=0$, so there is no membrane energy and the first three equations in (3.2) hold trivially. Upon rescaling the coordinates as $x \hookrightarrow t x, y \hookrightarrow k^{-1} y$ and the amplitude of $\theta$ consistently with that of $\psi$ as $\theta \hookrightarrow k t \theta$, writing $r=k t$, and finally scaling the energy density by a factor $48(1-\nu)^{-1}$, the resulting scaled shear \& bending energy density of the short-range layer mode takes the form

$$
\begin{aligned}
f_{s b}(\mathbf{u}) & =12 \gamma\left(r^{2} \theta^{2}+\psi^{2}\right) \\
& +2 r^{2} \nu(1-\nu)^{-1}\left(\frac{\partial \theta}{\partial x}+\frac{\partial \psi}{\partial y}\right)^{2}+2 r^{2}\left(\frac{\partial \theta}{\partial x}\right)^{2}+2 r^{2}\left(\frac{\partial \psi}{\partial y}\right)^{2} \\
& +\left(r^{2} \frac{\partial \theta}{\partial y}+\frac{\partial \psi}{\partial x}\right)^{2} \quad(n=1) .
\end{aligned}
$$

\subsection{Asymptotics of the layer modes}

We observe from (5.2) and (5.4)-(5.7) that some of the coefficients in these energy expressions tend to infinity when $t / L \rightarrow 0$ or $r \rightarrow 0$. The expressions being scaled so that the dominant terms have coefficient $\sim 1$, this means that the energy formulation of the layer mode problem becomes a constrained minimization problem at the mentioned parametric limits. In view of (5.2) and (5.4)-(5.7), the constraints that are enforced in this way are the following:

$$
\begin{array}{cc}
\theta-\frac{\partial w}{\partial x}=0 & (n=2,3,4), \\
\psi-\frac{\partial w}{\partial y}=0 \quad & (n=3,4), \\
\frac{\partial v}{\partial x}+2 c w=0 & (n=3), \\
\frac{\partial u}{\partial x}+a w=0 \quad(n=4), \\
\frac{\partial u}{\partial y}+\frac{\partial v}{\partial x}=0 \quad(n=4) .
\end{array}
$$


Note that (5.9), (5.10) are the usual shear constraints (Kirchhoff-Love constraints) of classical plate \& shell theory. In case of the layer modes, these constraints are enforced anisotropically so that the constraint (5.9) in the direction of the decay is stronger, see (5.2). When $n=2$, one has $(L / t)^{2} r^{2}=(R k)^{2} r^{4-2 n}=(R k)^{2}$ in $(5.2)$, so in this case the tangential shear constraint (5.10) would only be enforced at the limit $R k=\infty$.

When $n=2$, the energy formulation enforces shear constraints only. The additional constraints (5.11)-(5.13) that appear in case of hyperbolic or parabolic degeneration we name membrane constraints. In view of (5.6)-(5.7), these constraints are enforced at $r=0$.

Remark 5.2 In (5.9)-(5.13) we have listed only the kinematic constraints that are enforced asymptotically because some coefficients in the energy expression tend to infinity. Other constraints that arise from the Euler equations (i.e., from the static equilibrium) we consider static. In case $n=2$ the following static constraints hold at $t / L=0, r=0$ (see also Remark 5.3 below):

$$
\begin{array}{rlrl}
\psi-\frac{\partial w}{\partial y}=0 & (n=2) \\
\frac{\partial u}{\partial x}+(a+\nu b) w=0 & & (n=2, a+\nu b \neq 0), \\
\frac{\partial v}{\partial x}+2 c w=0 & & (n=2, c \neq 0) .
\end{array}
$$

We note that, unlike (5.9)-(5.13), these constraints are harmless from the finite element modelling point of view (see Section 5.3 below).

Remark 5.3 From the scaling of the membrane strains terms $\beta_{11}+\nu \beta_{22}$ and $\beta_{12}$ above we see that these terms actually give an asymptotically vanishing contribution to the membrane energy of any of the main layer modes. Namely, the contribution due to $\beta_{11}+\nu \beta_{22}$ (the first terms in (5.4)-(5.7)) is of order $O\left(r^{4}\right)$ and that due to $\beta_{12}$ (the last terms) is of order $O\left(r^{2}\right)$. Thus the energy is concentrated on the strain component $\beta_{22}$ in (5.3) when $r \rightarrow 0$.

Remark 5.4 In the parametric range where $r \sim 1$ or $r \gg 1$, the shear constraints (5.9)(5.10) are still enforced, this time as kinematic constraints in all cases, and with equal strength when $t / L=k t \rightarrow 0$. No membrane constraints arise in this range (see Remark 5.1 above).

Remark 5.5 By studying the Euler equations of the scaled energy principle

$$
F(\mathbf{u})=\int_{0}^{\infty} \int_{0}^{2 \pi}\left(f_{s b}(\mathbf{u})+f_{m}(\mathbf{u})\right) d x d y=\min !
$$

and passing to the zero limits of parameters $t / L, r$, and $\epsilon$ in (5.2)-(5.8), we find that each displacement or rotation component of a given layer mode satisfies asymptotically a differential equation that is characteristic for the mode. In the scaled coordinates (as above), these 
characteristic equations are

$$
\begin{array}{ll}
n=1: & \frac{\partial^{2} \phi}{\partial x^{2}}-12 \gamma \phi=0 \\
n=2: & \frac{\partial^{4} \phi}{\partial x^{4}}+12\left(1-\nu^{2}\right) b^{2} \phi \\
n=3: & \frac{\partial^{6} \phi}{\partial x^{6}}+48\left(1-\nu^{2}\right) c^{2} \frac{\partial^{2} \phi}{\partial y^{2}}=0 \\
n=4: & \frac{\partial^{8} \phi}{\partial x^{8}}+12\left(1-\nu^{2}\right) a^{2} \frac{\partial^{4} \phi}{\partial y^{4}}=0
\end{array}
$$

as could be predicted also from the Fourier analysis of Section 3. For the related 'classicism', see [7].

\subsection{Finite element considerations}

The parametrized energy formulations of the previous subsection raise a number of issues related to finite element modelling. In the present context, let us only draw some immediate conclusions regarding the simplest finite element model where $u, v, w, \theta, \psi$ are all continuous and either piecewise linear functions on a triangular mesh or piecewise bilinear functions on a quadrilateral mesh. We think of the mesh as subdividing some fixed domain $\omega$, say the unit square, in the scaled coordinates $x, y$. Assuming that the mesh is the image of the scaling map as well, we need then to make the (in general, rather severe) assumption that the scaling does not produce mesh angles that are close to $\pi$ [2].

For the assumed simplest finite element model it is well known that any of the constraints (5.9)-(5.13) causes locking, or approximation failure. In other words, when imposed in the finite element space, each of these constraints alone causes the space to collapse to a trivial subspace where the approximation properties are lost. (For a more detailed discussion in the context of cylindrical shells, see [23].) Thus the first conclusion is that when approximating any of the main shell layer modes $(n=2,3,4)$, the simplest finite element scheme suffers from asymptotic locking, i.e., fails to converge asymptotically at the zero limits of the parameters $t / L$ and $r$. When $n=2$, the locking effect at $t / L=0$ may be characterized as shear locking. As was seen above, this is an anisotropic effect in the layer-aligned coordinates when $r \ll 1$. In the cases $n=3,4$, the shear locking is supplemented with additional (anisotropic) membrane locking effects at the limit $r=0$.

The second conclusion concerns the more realistic situation where the parameters $t$ and $k$ take some non-zero and finite values. Since the layer mode to be approximated is uniformly smooth in the scaled coordinates, one must then have convergence (under the mentioned maximal angle condition) when the scaled mesh spacing tends to zero. However, one also expects parametric error amplification to arise from the large coefficients in the scaled energy density. To be more precise, let us measure the error in the relative energy norm as

$$
e=\frac{\left\|\mathbf{u}-\mathbf{u}_{h}\right\|}{\|\mathbf{u}\|},
$$


where $\mathbf{u}$ is the scaled layer mode, $\mathbf{u}_{h}$ is the finite element approximation of $\mathbf{u}$ on a mesh with (scaled) mesh spacing $h$, and the energy norm $\|\cdot\|$ is defined by

$$
\|\mathbf{u}\|=\left\{\int_{\omega}\left[f_{m}(\mathbf{u})+f_{s b}(\mathbf{u})\right] d x d y\right\}^{1 / 2},
$$

where $f_{m}, f_{s b}$ are defined by $(5.2),(5.4)-(5.8)$. The standard finite element approximation theory (cf. [3]) then predicts that

$$
e \sim K h, \quad K \sim\left(\sum_{i} C_{i}\right)^{1 / 2}
$$

where $C_{i}$ denotes the parametric coefficient multiplying the $i$ :th strain term in the scaled energy density. In the non-parametric situation where $C_{i} \sim 1$ for all $i$, this bound is not improvable [3], and we may predict that the bound is neither improvable (essentially) when some of the coefficients $C_{i}$ are large. Indeed, the approximation error must grow with $C_{i}$ unless there exists an interpolant that maintains (some of) its accuracy at $C_{i}=\infty$, i.e., under the corresponding constraint from the set (5.9)-(5.13).

Applying now the rule (5.14) in (5.2) and (5.4)-(5.7) we see that the largest coefficient $C_{i} \sim(L / t)^{2}$ arises from the shear strain $\rho_{1}$ in the direction of the decay of the layer, so we predict from (5.14) that when resolving a layer that decays in the length scale $L$, the nonparametric finite element error is amplified roughly by factor $K \sim L / t$. This rule thus predicts error amplification for all the main layer modes, the effect being the worse the larger the length scale $L$. The rule applies also to the short-range layer $(n=1, K \sim 1)$, since in that case no large coefficients appear in the scaled energy formulation (5.8).

Let us finally note that, in view of (5.4)-(5.7), the membrane locking alone causes error amplification by factor $K_{m} \sim r^{2-n}$ for the main layer modes. In the parametric range where these modes are true layer modes (see Section 4 above), the membrane locking effect is somewhat milder than the dominant shear locking. For example, when the layer mode varies smoothly in the curvature length scale $R$ along the layer generating line (as corresponding to $k \sim R^{-1}$ in the above analysis), the error amplification factors arising from the shear $(K)$ and membrane $\left(K_{m}\right)$ effects are

$$
K \sim\left(\frac{R}{t}\right)^{1-\frac{1}{n}}, \quad K_{m} \sim\left(\frac{R}{t}\right)^{1-\frac{2}{n}} \quad\left(n=2,3,4 ; k \sim R^{-1}\right) .
$$

These factors are expected, e.g., in the model problems shown in Figure 3 (for $n=2,4$ ) and in Figure 2 (for $n=2,3$ ).

Remark 5.6 We may compare (5.15) with the worst known error amplification that occurs when resolving smooth (in the length scale $R$ ) bending-dominated, or nearly inextensional deformations. In that case one has (see [23, 24])

$$
\left.K \sim K_{m} \sim \frac{R}{t} \quad \text { (inextensional mode, } k \sim R^{-1}\right) .
$$

We note that in our Fourier mode analysis, some of the long-range modes studied in Section 3.2 actually belong to this category, see [24] for more details in case of a cylindrical shell. 


\section{A Puiseux series expansion of the eigenvalues}

Case $R_{1}=R_{2}=R \quad \alpha=\frac{\pi}{4}$.

$$
\begin{aligned}
\lambda_{1,2,3,4}^{\mathrm{RN}}(t, R, k, \nu)= & \frac{\rho^{1 / 2}}{R^{1 / 2}} t^{-1 / 2}+\frac{1}{R^{3 / 2} \rho^{1 / 2}} \frac{(\nu+1)[(\nu-3) \gamma+2]+2 k^{2} R^{2} \gamma}{4 \gamma} t^{1 / 2}+O\left(t^{3 / 2}\right) \\
0 & \rho^{2}+12\left(1-\nu^{2}\right) \\
\lambda_{5,6}^{\mathrm{RN}}(t, R, k, \nu)= & k+\frac{1}{R^{3}}\left[\rho-\frac{k R}{48}\left(1-\nu^{2}\right)\right] t^{2}+O\left(t^{4}\right) \\
0= & 2304(\nu-1) \rho^{2}+96 k R(\nu-1)\left(\nu^{2}-1+4 k^{2} R^{2}\right) \rho \\
+ & k^{2} R^{2}\left[(\nu+1)\left(\nu^{4}-2 \nu^{3}+2 \nu+47\right)+8 k^{2} R^{2}(\nu-1)^{2}(\nu+1)+16 k^{4} R^{4}(\nu-1)\right] \\
\lambda_{7,8}^{\mathrm{RN}_{\mathrm{S}}}(t, R, k, \nu)= & -k+\frac{1}{R^{3}}\left[\rho+\frac{k R}{48}\left(1-\nu^{2}\right)\right] t^{2}+O\left(t^{4}\right) \\
0= & 2304(\nu-1) \rho^{2}-96 k R(\nu-1)\left(\nu^{2}-1+4 k^{2} R^{2}\right) \rho \\
+ & k^{2} R^{2}\left[(\nu+1)\left(\nu^{4}-2 \nu^{3}+2 \nu+47\right)+8 k^{2} R^{2}(\nu-1)^{2}(\nu+1)+16 k^{4} R^{4}(\nu-1)\right] \\
\lambda_{9,10}^{\mathrm{RN}}(t, R, k, \nu)= & \rho t^{-1}+\frac{\rho}{48 R^{2}}\left[\frac{\left(3-\nu^{2}\right) \gamma+2 k^{2} R^{2}}{\gamma}+\nu^{2}-1\right] t \\
& -\frac{\rho}{1152 \gamma R^{4}}\left[\frac{1}{\gamma}\left(\gamma^{2} \nu^{2}+\gamma k^{2} R^{2}\left(41-8 \nu+\nu^{2}\right)+k^{4} R^{4}\right)+\left(1-\nu^{2}\right)\left(\gamma+k^{2} R^{2}\right)\right] t^{3} \\
& +O\left(t^{5}\right) \\
0= & \rho^{2}-12 \gamma
\end{aligned}
$$

Case $R_{1}=R_{2}=R \quad \alpha=\frac{\pi}{2}$.

$$
\begin{aligned}
\lambda_{1,2,3,4}^{\mathrm{RN}}(t, R, k, \nu) & =\frac{\rho^{1 / 2}}{R^{1 / 2}} t^{-1 / 2}+\frac{1}{R^{3 / 2} \rho^{1 / 2}} \frac{(\nu+1)(1-\gamma)+k^{2} R^{2} \gamma}{2 \gamma} t^{1 / 2}+O\left(t^{3 / 2}\right) \\
0 & =\rho^{2}+12\left(1-\nu^{2}\right) \\
\lambda_{5,6}^{\mathrm{RN}_{\mathrm{S}}}(t, R, k, \nu)= & -k+\frac{\rho}{R} t^{2}+O\left(t^{4}\right) \\
0= & 144 R^{2}(\nu-1) \rho^{2}+24 k^{3} R^{3}(\nu-1) \rho+k^{6} R^{4}(\nu-1)-3 k^{2}(\nu+1) \\
\lambda_{7,8}^{\mathrm{RN}}(t, R, k, \nu)= & k+\frac{\rho}{R} t^{2}+O\left(t^{4}\right) \\
0= & 144 R^{2}(\nu-1) \rho^{2}-24 k^{3} R^{3}(\nu-1) \rho+k^{6} R^{4}(\nu-1)-3 k^{2}(\nu+1) \\
\lambda_{9,10}^{\mathrm{RN}}(t, R, k, \nu)= & \rho t^{-1}+\frac{\rho\left(4 \gamma+k^{2} R^{2}\right)}{24 \gamma R^{2}} t-\frac{\rho\left(16 \gamma^{2}-8 k^{2} R^{2} \gamma(\nu+6)+k^{4} R^{4}\right)}{1152 \gamma^{2} R^{4}} t^{3}+O\left(t^{5}\right) \\
0 & =\rho^{2}-12 \gamma
\end{aligned}
$$


Case $R_{1}=R \quad R_{2}=\infty \quad \alpha=\frac{\pi}{2}$

$$
\begin{aligned}
\lambda_{1,2,3,4}^{\mathrm{RN}}(t, R, k, \nu) & =\frac{\rho^{1 / 2}}{R^{1 / 2}} t^{-1 / 2}+\frac{\nu+1+2 \gamma k^{2} R^{2}}{2 \gamma \rho^{1 / 2} R^{3 / 2}} t^{1 / 2}+O\left(t^{3 / 2}\right) \\
0 & =\rho^{2}+12\left(1-\nu^{2}\right) \\
\lambda_{5,6,7,8}^{\mathrm{RN}}(t, R, k, \nu) & =-\frac{1}{12\left(1-\nu^{2}\right) R^{1 / 2} \rho^{3 / 2}} k\left(1-k^{2} R^{2}\right)^{2} t^{1 / 2}-\frac{k\left(1-k^{2} R^{2}\right)^{4}}{144\left(1-\nu^{2}\right)^{2} R^{3 / 2} \rho^{5 / 2}} t^{3 / 2}+O\left(t^{5 / 2}\right) \\
0 & =12\left(1-\nu^{2}\right) \rho^{2}+\left(1-k^{2} R^{2}\right)^{2} \\
\lambda_{9,10}^{\mathrm{RN}}(t, R, k, \nu) & =\rho t^{-1}+\frac{\rho}{24 \gamma R^{2}}\left(4 \gamma+k^{2} R^{2}\right) t \\
& -\frac{\rho}{1152 \gamma^{2} R^{4}}\left(16 \gamma^{2}-8 \gamma k^{2} R^{2}(\nu+6)+k^{4} R^{4}\right) t^{3}+O\left(t^{5}\right) \\
0 & =\rho^{2}-12 \gamma
\end{aligned}
$$

Case $R_{1}=R \quad R_{2}=-R \quad \alpha=\frac{\pi}{4}$

$$
\begin{aligned}
\lambda_{1,2,3,4,5,6}^{\mathrm{RN}}(t, R, k, \nu)= & \frac{\rho^{1 / 3}}{R^{1 / 3}} t^{-1 / 3}+\frac{\nu-3+2 k^{2} R^{2}}{3 \rho^{1 / 3} R^{5 / 3}} t^{1 / 3} \\
& -\frac{\left(1-\nu^{2}\right) \gamma+k^{2} R^{2}[8(\nu+1)+2 \gamma(5+\nu)]+2 \gamma k^{4} R^{4}}{6 \rho \gamma R^{3}} t+O\left(t^{5 / 3}\right) \\
0= & \rho^{2}-48 k^{2}\left(1-\nu^{2}\right) \\
\lambda_{7,8}^{\mathrm{RN}_{\mathrm{S}}}(t, R, k, \nu)= & \frac{\rho}{R} t+\frac{\rho}{R^{3}}\left\{\frac { 1 } { 4 8 } \left[\left(2 \gamma\left(-3 \nu^{4}+20 \nu^{3}-28 \nu^{2}+8 \nu-5\right)\right.\right.\right. \\
+ & 2 k^{2} R^{2}\left(2\left(9+3 \nu-5 \nu^{2}+\nu^{3}\right)+\gamma\left(-2+14 \nu+7 \nu^{2}-8 \nu^{3}+\nu^{4}\right)\right) \\
+ & 2 k^{4} R^{4}\left(4(\nu+1)(\nu-3)+\gamma\left(12-16 \nu+\nu^{2}+\nu^{3}\right)\right) \\
+ & \left.\left.k^{6} R^{6}(4(1+\nu)+\gamma(7 \nu-13))+2 \gamma k^{8} R^{8}\right)\right] \\
& \left(\gamma\left(1-\nu^{2}\right)\left[2(3 \nu-5)+2(3-\nu) k^{2} R^{2}-k^{4} R^{4}\right]\right) \\
& \\
& \left.-\frac{1}{24}(\nu+1)\right\} t^{3}+O\left(t^{5}\right) \\
0 & -48\left(1-\nu^{2}\right) \rho^{2}+k^{2}\left[2(5-3 \nu)+2 k^{2} R^{2}(\nu-3)+k^{4} R^{4}\right] \\
= & \rho t^{-1}+\frac{\rho}{R^{2}}\left[\frac{(3+\nu) \gamma+2 k^{2} R^{2}}{48 \gamma}-\frac{1}{24}(\nu+1)\right] t+O\left(t^{3}\right) \\
= & \rho^{2}-12 \gamma \\
\lambda_{9,10}^{\mathrm{RN}}(t, R, k, \nu) &
\end{aligned}
$$


Case $R_{1}=R \quad R_{2}=\infty \quad \alpha=0$

$$
\begin{aligned}
\lambda_{1, \ldots, 8}^{\mathrm{RN}}(t, R, k, \nu) & =\frac{\rho^{1 / 4} k^{1 / 2}}{R^{1 / 4}} t^{-1 / 4}+\frac{-1+2 k^{2} R^{2}}{4 \rho^{1 / 4} k^{1 / 2} R^{7 / 4}} t^{1 / 4}-\frac{-1+12 k^{2} R^{2}+4 k^{4} R^{4}}{32 \rho^{3 / 4} k^{3 / 2} R^{13 / 4}} t^{3 / 4} \\
& +\frac{\left[32(1+\nu) k^{4} R^{4}+\gamma\left(1+10 k^{2} R^{2}+4\left(1+8 \nu^{2}\right) k^{4} R^{4}+8 k^{6} R^{6}\right)\right]}{128 \gamma \rho^{5 / 4} k^{5 / 2} R^{19 / 4}} t^{5 / 4} \\
& +O\left(t^{7 / 4}\right) \\
0 & =\rho^{2}+12\left(1-\nu^{2}\right) \\
\lambda_{9,10}^{\mathrm{RN}}(t, R, k, \nu) & =\rho t^{-1}+\frac{\rho}{24 \gamma} k^{2} t-\frac{\rho}{1152 \gamma^{2}} k^{4} t^{3}+O\left(t^{5}\right) \\
0 & =\rho^{2}-12 \gamma
\end{aligned}
$$

\section{References}

[1] D.N. Arnold and R.S. Falk, Asymptotic analysis of the boundary layer for the ReissnerMindlin plate model, SIAM J. Math. Anal. 27 (1996) 486-514.

[2] I. Babuška and A.K. Aziz, On the angle condition in the finite element method, SIAM J. Numer. Anal. 13 (1976) 214-226.

[3] D. Braess, Finite Elements, Cambridge University Press 1997.

[4] P.G. Ciarlet, Introduction to Linear Shell Theory, Gauthier-Villars 1998.

[5] W. Flügge, Statik und Dynamik der Schalen, Springer 1934.

[6] W. Flügge, Stresses in Shells, 2nd ed., Springer 1973.

[7] A.L. Gol'denveizer, Theory of Thin Elastic Shells, Pergamon 1961. Transl. of the original Russian vol.: Gostekhizdat 1953.

[8] K. Gerdes, A.M. Matache and C. Schwab, On membrane locking in $h p$ FEM for a cylindrical shell, Zeitschrift für Angewandte Mathematik und Mechanik 78 (1998) 663-686.

[9] H. Hakula, Y. Leino and J. Pitkäranta, Scale resolution, locking, and high-order finite element modelling of shells, Comput. Meth. Appl. Mech. Engrg. 133 (1996) 157-182.

[10] H. Hakula and J. Pitkäranta, Pinched shells of revolution: Experiments on high-order FEM, Proc. of the Third International Conference of spectral and high-order methods (ICOSAHOM95, Houston 1995) 193-201.

[11] W.T. Koiter, A consistent first approximation in the general theory of thin elastic shells, Proc. IUTAM Symp. Theory of Thin Shells (Delft 1959), North-Holland 1960, 12-33. 
[12] W.T. Koiter, On the foundations of the linear theory of thin elastic shells, Proc. Kon. Nederl. Akad. Wetensch., B 73 (1970) 169-195.

[13] A.E.H. Love, The small free vibrations and deformation of a thin elastic shell, Phil. Trans. R. Soc. 179 a (1888) 491-546.

[14] A.E.H. Love, A Treatise on the Mathematical Theory of Elasticity, 4th ed., Cambridge University Press 1927.

[15] S. Lukasiewicz, Local Loads in Plates and Shells, Sijthoff \& Noordhoff 1979.

[16] P.M. Naghdi, On the theory of thin elastic shells, Quart. Appl. Math. 14 (1957) 369-380.

[17] P.M. Naghdi, Foundations of elastic shell theory, in Progress in Solid Mechanics, Vol. 4, I.N. Sneddon and R. Hill, eds., North-Holland 1963, 1-90.

[18] F.I. Niordson, ed., Theory of Thin Shells, Proc. Second IUTAM Symp. (Copenhagen 1967), Springer 1969.

[19] V.V. Novozhilov, The Theory of Thin Shells, Noordhoff 1959. Transl. of the first Russian ed.: Oborongiz 1951.

[20] J. Piila, Characterization of the membrane theory of a clamped shell. The elliptic case, Math. Models Methods Appl. Sci. $\left(M^{3} A S\right) 4$ (1994) 147-177.

[21] J. Piila, Characterization of the membrane theory of a clamped shell. The hyperbolic case, Math. Models Methods Appl. Sci. $\left(M^{3} A S\right) 6$ (1996) 169-194.

[22] J. Piila and J. Pitkäranta, Characterization of the membrane theory of a clamped shell. The parabolic case, Math. Models Methods Appl. Sci. $\left(M^{3} A S\right) 3$ (1993) 417-442.

[23] J. Pitkäranta, The problem of membrane locking in finite element analysis of cylindrical shells, Numer. Math. 61 (1992) 523-542.

[24] J. Pitkäranta, Y. Leino, O. Ovaskainen and J. Piila, Shell deformation states and the finite element method: A benchmark study of cylindrical shells, Comput. Meth. Appl. Mech. Engrg. 128 (1995) 81-121.

[25] J. Pitkäranta and E. Sanchez-Palencia, On the asymptotic behaviour of sensitive shells with small thickness, Compt. Rend. Acad. Sci. Paris, Série II b, 325 (1997) 127-134.

[26] E. Reissner, The effect of transverse shear deformation on the bending of elastic plates, J. Appl. Mech. 12 (1945) A68-A77.

[27] E. Reissner, Stress-strain relations in the theory of thin elastic shells, J. Math. Phys. 31 (1952) 109-119.

[28] H.S. Rutten, Asymptotic approximation in the three-dimensional theory of thin and thick elastic shells, in: F.I. Niordson, ed., Theory of Thin Shells, Springer 1969, 115-134. 
[29] H.S. Rutten, Theory and Design of Shells on the Basis of Asymptotic Analysis, Rutten \& Kruisman, Consulting Engineers, Holland 1974.

[30] É. Sanchez-Palencia, Statique et dynamique des coques minces, II: Cas de flexion pure inhibée, approximation membranaire, Compt. Rend. Acad. Sci. Paris, Série I, 309 (1989) $531-537$.

[31] É. Sanchez-Palencia, On the membrane approximation of thin elastic shells in the hyperbolic case, Revista Mathematica de la Universidad Complutense de Madrid, Vol. 6, No. 2 (1993).

[32] J. Sanchez-Hubert and É. Sanchez-Palencia, Coques Élastiques Minces. Propriétés Asymptotiques, Masson 1997.

[33] Theory of Shells and Plates, Proc. of the 4th All-Union Conf. (Erevan 1962), Israel Program for Sci. Transl. 1966.

[34] J.R. Vinson, Structural Mechanics: The Behavior of Plates and Shells, Wiley 1974.

[35] W.S. Wlassow (V.Z. Vlasov), Allgemeine Schalentheorie und ihre Anwendung in der Technik, Berlin Akademie 1958. Transl. of the original Russian vol.: Gostekhizdat 1949. 